\title{
Frozen Gaussian Approximation-Based Two-level Methods for Multi-frequency Time Dependent Schrödinger Equation
}

\author{
E. Lorin* \\ School of Mathematics and Statistics, Carleton University, Ottawa, Canada K1S 5B6. \\ Centre de Recherches Mathématiques, Université de Montréal, Montréal, Canada, H3T 1J4 \\ $\mathrm{Xu}$ Yang \\ University of California at Santa Barbara, Department of Mathematics, CA 93106, USA
}

\begin{abstract}
In this paper, we develop two-level numerical methods for the time-dependent Schrödinger equation (TDSE) in multi-frequency regimes. This work is motivated by attosecond science [P.B. Corkum and F. Krausz, Nature Physics, 3 (2007), 381-387], which refers to the interaction of short and intense laser pulses with quantum particles generating wide frequency spectrum light and allowing for the coherent emission of attosecond pulses ( 1 attosecond $=10^{-18}$ second). The principle of the proposed methods consists in decomposing a wavefunction into a low/moderate frequency (quantum) contribution, and a high frequency contribution exhibiting a semi-classical behavior. Low/moderate frequencies are computed through the direct solution of the quantum TDSE on a coarse mesh, and the high frequency contribution is computed by frozen Gaussian approximation [M.F. Herman and E. Kluk, Chem. Phys., 91 (1984), 27-34]. This paper is devoted to the derivation of consistent, accurate and efficient algorithms performing such a decomposition and the time evolution of the wavefunction in the multi-frequency regime. Numerical simulations are provided to illustrate the accuracy and efficiency of derived algorithms.
\end{abstract}

Keywords: Geometric optics, frozen Gaussian approximation, Schrödinger equation, multilevel method, attosecond science

\section{Introduction}

\subsection{Introductory remarks}

In this paper, we are interested in developing two-level methods for solving numerically the timedependent Schrödinger equation (TDSE) modeling in particular, the interaction of intense electric fields with quantum particles in attosecond science [5]. In $\Omega \subseteq \mathbb{R}^{3}$ and under the Born-Oppenheimer and dipole approximations [4], the TDSE reads in length gauge [3]

$$
\left\{\begin{array}{llll}
\mathrm{i} \partial_{t} \psi(t, \mathbf{x}) & =-\frac{1}{2} \Delta \psi(t, \mathbf{x})+\mathbf{x} \cdot \mathbf{E}(t) \psi(t, \mathbf{x})+V(\mathbf{x}) \psi(t, \mathbf{x}), & & (t, \mathbf{x}) \in(0, T) \times \Omega \\
\psi(0, \mathbf{x}) & =\phi_{0}(\mathbf{x}), & & \mathbf{x} \in \Omega \\
\psi(t, \mathbf{x}) & =0, & & (t, \mathbf{x}) \in(0, T) \times \partial \Omega
\end{array}\right.
$$

where $V$ is a space-dependent (nuclear) potential, and $\mathbf{E}$ denotes the external electric field the quantum particle is subject to. It is well-known that the interaction of short and intense electromagnetic fields with

\footnotetext{
*Corresponding author

Email addresses: elorin@math.carleton.ca (E. Lorin*), xuyang@math.ucsb.edu (Xu Yang)
}

Preprint submitted to Computer Physics Communications

May 21, 2016 
atoms or molecules leads to complex nonlinear phenomena among which, one of the most important is the generation of high frequency photons, see $[3,1,2]$. Let us start from a localized state

$$
H_{0} \phi_{0}=\lambda_{0} \phi_{0}
$$

where $\lambda_{0}$ (resp. $\phi_{0}$ ) is the smallest eigenvalue (resp. eigenfunction) of the field-free atomic Schrödinger Hamiltonian $H_{0}=-\triangle / 2+V$. We consider a laser field $\mathbf{E}(t)=E(t) \mathbf{e}_{x}$ of frequency $\omega_{0}$ and intensity $E_{0}^{2}$, interacting with the atom (or the molecule), where $\mathbf{e}_{x}$ stands for the unit direction in $x$. Subject to such a field, the atom/molecule is first ionized, then the free electrons gain ponderomotive energy $e^{2} E_{0}^{2} / 4 m \omega_{0}^{2}$, where $e$ is the electron charge and $m$ is the nucleus mass. In a third stage, electrons are recombined with their parent ion and generate high order harmonics (HHG) by multi-photon ionization. This process is described for the hydrogen atom in the celebrated paper [10], where strong-field-approximation is used to derive an accurate 3-step HHG model, using Newton's law coupled with a tunneling model. It is shown in [10] that after the laser-hydrogen interaction, the time harmonic spectrum is constituted by a plateau, up to a cut-off frequency $\omega_{c}$, such that $\hbar \omega_{c}=N_{c} \omega_{0} \hbar \approx 3.17 U_{p}+I_{p}$, where $N_{c} \in 2 \mathbb{N}^{*}+1$ is the cut-off frequency order, and $I_{p}$ is the ionization potential. Starting from a one-frequency problem (associated to the incoming pulse frequency), high time- and space-frequencies (wavenumbers or Fourier modes) are then generated. Appropriate numerical methods should tackle this multiscale problem, and deal simultaneously with quantum and semi-classical regimes. This paper is dedicated to the derivation of two-level methods for this multiscale problem. The principle is to rigorously couple usual real space methods for relatively low space-and-time frequency regime, with a geometric optics-type method, the frozen Gaussian approximation (FGA), for high space-and-time frequency regime. What makes this problem particularly difficult is that high frequencies are dynamically generated. Fortunately, we can benefit from the 3-step model which provides quite relevant and precise information on i) when the multi-photon ionization occurs, as well as ii) the recombination energy, which is related to the highest generated frequencies. This is a very relevant information which allows us to determine if and when, the FGA should be reinitialized. Between these times, which can accurately be numerically evaluated, we can solve the Schrödinger equation on two separate frequency levels i) one solving the Schrödinger equation in the quantum regime on a coarse grid, ii) one solving the Schrödinger equation in the semi-classical regime, using a FGA, which provides an accurate and cheap (compared to a full quantum computation on very fine grid) computation of the TDSE solution on wide frequency range. We refer to $[8,16,6,9]$ for numerical methods for quantum and classical wave equations in the semi-classical regime.

The paper is organized as follows. Subsection 1.2 is dedicated to the presentation of the frozen Gaussian approximation (FGA) and the discretization for the TDSE in the semi-classical regime. Corkum's threestep model is presented in 2. Section 3.2 (resp. 3.3) is devoted to the two-level method without (resp. with) dynamical generation of high frequency. Numerical experiences are presented in Section 4 . We finally conclude in Section 5.

\subsection{Frozen Gaussian approximation for TDSE in semi-classical regime}

In this section, we describe the numerical evolution of the TDSE wavefunction, in the semi-classical regime using the FGA and denoted by $\psi_{\mathrm{FGA}}$.

FGA formulation. We here recall the main steps to construct a FGA. This method was originally developed by Herman-Kluk (HK) [7] and was later mathematically analyzed in [17]. More recently, the HK formalism was used and analyzed to derive fast numerical solvers in the semi-classical regime for different classes of partial differential equations: the Schrödinger equation [18], the classical wave equation [14, 13] and linear hyperbolic systems of conservation laws [15]. FGA was also used to accelerate the convergence of Schwarz Waveform Relaxation domain decomposition algorithms [12]. FGA allows for the computational evolution of high frequency wavefunction. More specifically, we start in $d$-dimensions, from

$$
\mathrm{i} \frac{\partial \psi}{\partial t}(t, \mathbf{x})=-\frac{1}{2} \triangle \psi(t, \mathbf{x})+W(t, \mathbf{x}) \psi(t, \mathbf{x}), \mathbf{x} \in \mathbb{R}^{d}, t \in(0, T),
$$


where $\psi(0, \mathbf{x})=\phi(\mathbf{x}) \in L^{2}\left(\mathbb{R}^{d}, \mathbb{C}\right)$. We rescale the TDSE as follows. For $\varepsilon \ll 1$, we set $t^{\prime}=\varepsilon t, \mathbf{x}^{\prime}=\varepsilon \mathbf{x}$, and we get

$$
\mathbf{i} \varepsilon \frac{\partial \psi^{\varepsilon}}{\partial t^{\prime}}\left(t^{\prime}, \mathbf{x}^{\prime}\right)=-\frac{\varepsilon^{2}}{2} \triangle \psi^{\varepsilon}\left(t, \mathbf{x}^{\prime}\right)+W^{\varepsilon}\left(t^{\prime}, \mathbf{x}^{\prime}\right) \psi^{\varepsilon}\left(t^{\prime}, \mathbf{x}^{\prime}\right), \mathbf{x}^{\prime} \in \mathbb{R}^{d}, t^{\prime} \in(0, \varepsilon T),
$$

with $\psi^{\varepsilon}\left(0, \mathbf{x}^{\prime}\right)=\psi_{0}^{\varepsilon}\left(\mathbf{x}^{\prime}\right):=\phi_{0}(\varepsilon \mathbf{x}) \in L^{2}\left(\mathbb{R}^{d}, \mathbb{C}\right)$ and $W^{\varepsilon}\left(t^{\prime}, \mathbf{x}^{\prime}\right)=W(\varepsilon \mathbf{x}, \varepsilon t)$. FGA is computed at $\left(t^{\prime}, \mathbf{x}^{\prime}\right)$, that is $\psi_{\mathrm{FGA}}\left(t^{\prime}, \mathbf{x}^{\prime}\right)=\psi_{\mathrm{FGA}}(\varepsilon t, \varepsilon \mathbf{x})$ with $t^{\prime} \in(0, \varepsilon T)$. FGA reads for $t^{\prime}>0$

$$
\psi_{F G A, \varepsilon}\left(t^{\prime}, \mathbf{x}^{\prime}\right)=\frac{1}{(2 \pi \varepsilon)^{3 d / 2}} \int_{\mathbb{R}^{d} \times B\left(\mathbf{0}_{d}, \varepsilon^{-1}\right)} a\left(t^{\prime}, \mathbf{q}, \mathbf{p}\right) e^{\frac{i}{\varepsilon} \Phi\left(t^{\prime}, \mathbf{x}^{\prime}, \mathbf{y}^{\prime}, \mathbf{q}, \mathbf{p}\right)} \psi_{0}^{\varepsilon}\left(\mathbf{y}^{\prime}\right) d \mathbf{y}^{\prime} d \mathbf{p} d \mathbf{q},
$$

with

$$
\begin{aligned}
\Phi\left(t^{\prime}, \mathbf{x}^{\prime}, \mathbf{y}^{\prime}, \mathbf{q}, \mathbf{p}\right)= & S\left(t^{\prime}, \mathbf{q}, \mathbf{p}\right)+\frac{i}{2}\left|\mathbf{x}^{\prime}-\mathbf{Q}\left(t^{\prime}, \mathbf{q}, \mathbf{p}\right)\right|^{2}+\mathbf{P}\left(t^{\prime}, \mathbf{q}, \mathbf{p}\right) \cdot\left(\mathbf{x}^{\prime}-\mathbf{Q}\left(t^{\prime}, \mathbf{q}, \mathbf{p}\right)\right) \\
& +\frac{i}{2}\left|\mathbf{y}^{\prime}-\mathbf{q}\right|^{2}-\mathbf{p} \cdot\left(\mathbf{y}^{\prime}-\mathbf{q}\right),
\end{aligned}
$$

where the Hamiltonian reads

$$
H\left(t^{\prime}, \mathbf{Q}, \mathbf{P}\right)=\frac{|\mathbf{P}|^{2}}{2}+W\left(t^{\prime}, \mathbf{Q}\right),
$$

with $W(t, \mathbf{Q})=V(\mathbf{Q})+\mathbf{Q} \cdot \mathbf{E}\left(t^{\prime}\right)$ and the Hamiltonian flow satisfies

$$
\begin{cases}\frac{d \mathbf{Q}}{d t^{\prime}}=\mathbf{P}, & \mathbf{Q}(0, \mathbf{q}, \mathbf{p})=\mathbf{q} \\ \frac{d \mathbf{P}}{d t^{\prime}}=-\partial_{\mathbf{Q}} W\left(t^{\prime}, \mathbf{Q}\right), & \mathbf{P}(0, \mathbf{q}, \mathbf{p})=\mathbf{p}\end{cases}
$$

The classical action function satisfies

$$
\frac{d S}{d t^{\prime}}=\frac{|\mathbf{P}|^{2}}{2}-W\left(t^{\prime}, \mathbf{Q}\right)
$$

and the amplitude

$$
\frac{d a}{d t^{\prime}}\left(t^{\prime}, \mathbf{q}, \mathbf{p}\right)=\frac{1}{2} a\left(t^{\prime}, \mathbf{q}, \mathbf{p}\right) \operatorname{Tr}\left(\mathbf{Z}^{-1}\left(\partial_{\mathbf{z}} \mathbf{P}\left(t^{\prime}, \mathbf{q}, \mathbf{p}\right)-i \partial_{\mathbf{z}} \mathbf{Q} \partial_{\mathbf{q}}^{2} \mathbf{Q}\left(t^{\prime}, \mathbf{q}, \mathbf{p}\right)\right)\right)
$$

where $\partial_{\mathbf{z}}=\partial_{\mathbf{q}}-i \partial_{\mathbf{p}}$ and $\mathbf{Z}=\partial_{\mathbf{z}}(\mathbf{Q}+i \mathbf{P})$.

Discretization of FGA. The discretization of FGAs is extensively discussed in the literature [18, 15, 14, 13]. We denote by $(\mathbf{j}, \mathbf{k}, \mathbf{l})$ the elements of the index sets denoted by $D_{\boldsymbol{x}} \times D_{\mathbf{q}} \times \widetilde{D}_{\mathbf{p}}{ }^{1}$, then the FGA reads as, at time $t_{n}^{\prime}$ and discretization nodes denoted $\boldsymbol{x}_{\mathbf{j}}$,

$$
\begin{aligned}
& \phi_{\mathrm{FGA}}^{\varepsilon}\left(t_{n}^{\prime}, \boldsymbol{x}^{\mathbf{j}}\right)= \\
& \quad \sum_{(\mathbf{k}, \mathbf{l}) \in D_{\mathbf{q}} \times \widetilde{D}_{\mathbf{p}}} \frac{a_{\mathbf{k}, \mathbf{l}}\left(t_{n}^{\prime}\right) r_{\theta}\left(\left|\mathbf{x}^{\mathbf{j}}-\mathbf{q}_{\mathbf{k}}\right|\right)}{(2 \pi \varepsilon)^{3 d / 2}} w^{\varepsilon}\left(\mathbf{q}_{\mathbf{k}}, \mathbf{p}_{\mathbf{k}, \mathbf{l}}\right) e^{\frac{i}{\varepsilon}\left(S_{\mathbf{k}, \mathbf{l}}\left(t_{n}^{\prime}\right)+\mathbf{P}_{\mathbf{k}, \mathbf{l}}\left(t_{n}^{\prime}\right) \cdot\left(\boldsymbol{x}^{\mathbf{j}}-\mathbf{Q}_{\mathbf{k}, \mathbf{l}}\left(t_{n}^{\prime}\right)\right)\right)-\frac{1}{2 \varepsilon}\left|\boldsymbol{x}^{\mathbf{j}}-\mathbf{Q}_{\mathbf{k}, \mathbf{l}}\left(t_{n}^{\prime}\right)\right|^{2}}|\delta \mathbf{q}||\delta \mathbf{p}|,
\end{aligned}
$$

\footnotetext{
${ }^{1}$ The notation $\widetilde{D}_{\mathbf{p}}$ is used to precise that the integrand in the FGA is usually localized with respect to $\mathbf{p}$, see $[18,15]$.
} 
where $a_{\mathbf{k}, \mathbf{l}}\left(t_{n}^{\prime}\right)\left(\right.$ resp. $\left.\mathbf{P}_{\mathbf{k}, \mathbf{l}}\left(t_{n}^{\prime}\right), \mathbf{Q}_{\mathbf{k}, \mathbf{l}}\left(t_{n}^{\prime}\right)\right)$ denotes the approximation of $a$ in (3) (resp. $\mathbf{P}, \mathbf{Q}$ in $\left.(2)\right)$ at $\left(\mathbf{q}_{\mathbf{k}}, \mathbf{p}_{\mathbf{k}, \mathbf{l}}\right)$ at time $t_{n}^{\prime}, \delta \mathbf{q}$ and $\delta \mathbf{p}$ denote the elementary increment in $\mathbf{q}$ and $\mathbf{p}$, and $r_{\theta}$ is the local truncation function with a radius $\theta[18]$. In addition, for any $\mathbf{k} \in D_{\mathbf{q}}$ and $\mathbf{l} \in D_{\mathbf{p}}$,

$$
w^{\varepsilon}\left(\mathbf{q}_{\mathbf{k}}, \mathbf{p}_{\mathbf{k}, \mathbf{l}}\right)=\sum_{\mathbf{j} \in D_{\mathbf{x}}} e^{\frac{i}{\varepsilon}\left(-\mathbf{p}_{\mathbf{k}, 1} \cdot\left(\mathbf{y}^{\mathbf{j}}-\mathbf{q}_{\mathbf{k}}\right)+\frac{i}{2}\left|\mathbf{y}^{\mathbf{j}}-\mathbf{q}_{\mathbf{k}}\right|^{2}\right)} \phi_{0}\left(\mathbf{y}_{\mathbf{j}}\right) r_{\theta}\left(\left|\mathbf{y}^{\mathbf{j}}-\mathbf{q}_{\mathbf{k}}\right|\right)|\delta \mathbf{y}| .
$$

Then $\left(\mathbf{Q}_{\mathbf{k}, \mathbf{l}}\left(t_{n}^{\prime}\right), \mathbf{P}_{\mathbf{k}, \mathbf{l}}\left(t_{n}^{\prime}\right)\right)$ and $a_{\mathbf{k}, \mathbf{l}}\left(t_{n}^{\prime}\right)$ are updated using a fourth order Runge-Kutta (RK4) scheme, for solving at time $t_{n}^{\prime}$ :

$$
\left\{\begin{array}{l}
d \mathbf{Q}_{\mathbf{k}, \mathbf{l}} / d t^{\prime}=\mathbf{P}_{\mathbf{k}, \mathbf{l}} \\
d \mathbf{P}_{\mathbf{k}, \mathbf{l}} / d t^{\prime}=-\partial_{\mathbf{Q}} W_{\mathbf{k}, \mathbf{l}} \\
d a_{\mathbf{k}, \mathbf{l}} / d t^{\prime}=a_{\mathbf{k}, 1} \operatorname{Tr}\left(\mathbf{z}_{\mathbf{k}, \mathbf{l}}^{-1}\left(\partial_{\mathbf{z}} \mathbf{P}_{\mathbf{k}, \mathbf{l}}-i \partial_{\mathbf{z}} \mathbf{Q}_{\mathbf{k}, 1} \partial_{\mathbf{Q}}^{2} W_{\mathbf{k}, 1}\right)\right) / 2
\end{array}\right.
$$

In $1-\mathrm{d}, \mathbf{x}, \mathbf{q}, \mathbf{p}, \delta \mathbf{x}, \delta \mathbf{q}, \delta \mathbf{p}, \mathbf{i}, \mathbf{j}, \mathbf{k}$ is respectively denoted by $x, q, p, h, \delta q, \delta p, i, j, k$.

We recall that from [17], denoting by $\Psi_{e}$ the exact solution to the TDSE, there exists a positive constant $C(T)$ such that

$$
\sup _{t^{\prime} \in(0, \varepsilon T)}\left\|\Psi_{e}\left(t^{\prime}, \cdot\right)-\psi_{\mathrm{FGA}}\left(t^{\prime}, \cdot\right)\right\|_{L^{2}\left(\mathbb{R}^{d}\right)} \leqslant \varepsilon C(T)\left\|\phi_{0}\right\|_{L^{2}\left(\mathbb{R}^{d}\right)} .
$$

In the quantum regime, we will construct an approximate solution to (1), typically using a finite difference scheme. Denoting $\psi_{\mathrm{Q}}^{n}$ an approximation of $\psi_{\mathrm{Q}}\left(t_{n}, \cdot\right)$ on a coarse mesh at time $t_{n}$, we have

$$
\begin{aligned}
\mathrm{i} \frac{\psi_{\mathrm{Q}}^{n+1}-\psi_{\mathrm{Q}}^{n}}{\Delta t}= & -\frac{1}{4} \triangle_{h}\left(\psi_{\mathrm{Q}}^{n+1}+\psi_{\mathrm{Q}}^{n}\right)+\frac{1}{2} \mathbf{x} \cdot \mathbf{E}\left(t_{n}\right) \psi_{\mathrm{Q}}^{n} \\
& +\frac{1}{2} \mathbf{x} \cdot \mathbf{E}\left(t_{n+1}\right) \psi_{\mathrm{Q}}^{n+1}+\frac{1}{2} V\left(\mathbf{x}^{h}\right)\left(\psi_{\mathrm{Q}}^{n+1}+\psi_{\mathrm{Q}}^{n}\right),
\end{aligned}
$$

where $\triangle_{h}$ is a discrete Laplace operator.

\section{Three-step model}

We recall the main features of Corkum's three-step model [10] in one dimension, which will be useful for deriving the two-level method in the framework of field-particle interaction. Some numerical experiments will then be presented to illustrate the model. Although this model is well-known by laser physicists, this summary may be useful for the non-specialists.

\subsection{Three-step model: theory}

In the strong field approximation, it is assumed that a quantum particle subject to intense oscillating laser field is modeled by a classical+tunneling model as follows. We assume that an electron is initially localized at $x=x_{0}$ with velocity denoted by $v_{0}$. The ionization potential of the atom is denoted by $I_{p}$. In the classical mechanics framework, the dynamics of the electron of mass $m$ and charge $e$ is modeled by Newton's law: $m \ddot{x}(t)=-e E(t)$. The electric field $E$, assumed to be time-dependent only, is modeled by

$$
E(t)=E_{0} f(t) \cos \left(\omega_{0} t+\phi_{0}\right),
$$

where $f$ is the pulse envelope, $\phi_{0}$ the initial phase and $\omega_{0}$ the pulse frequency. In general $f$ is a Gaussian or $\sin ^{2}$ function [11], such as

$$
f(t)=1, \quad f(t)=\sin ^{2}\left(\pi \frac{T-t}{\alpha T}\right), \quad f(t)=\exp \left(-\alpha(t-T / 2)^{2}\right),
$$


where $T$ is the pulse duration and $\alpha$ some strictly positive constant. For instance, for $f(t)=1$ and $x_{0}=$ $v_{0}=0$, we easily get

$$
v(t)=\frac{E_{0}}{\omega_{0}}\left(\cos \left(\phi_{0}\right)-\cos \left(\omega_{0} t+\phi_{0}\right)\right), \quad x(t)=\frac{E_{0}}{\omega_{0}} \cos \left(\phi_{0}\right) t-\frac{E_{0}}{\omega_{0}^{2}}\left(\sin \left(\omega_{0} t+\phi_{0}\right)-\sin \left(\phi_{0}\right)\right) .
$$

In order to benefit from this model in our two-level method, we first define the times of return of the classical electron $t_{r} \in(0, T)$, such that $x\left(t_{r}\right)=x_{0}$, see [11]. There will be referred as classical recombination times and defined by

$$
\mathcal{T}_{r}=\left\{t_{r}^{(i)} \in(0, T): x\left(t_{r}^{(i)}\right)=x_{0}, i \in \mathbb{N}^{*}\right\} .
$$

In addition, it is possible to define the ponderomotive energy of the particle in the field, $U_{p}=e^{2} E_{0}^{2} / 4 m \omega_{0}^{2}$; see [10]. The energy of return at time, say $t=t_{r}$ is then defined by $I_{p}+m\left(\dot{x}\left(t_{r}\right)\right)^{2} / 2$. We consequently define

$$
\mathcal{E}_{r}=\left\{E_{r}^{(i)}=I_{p}+\frac{m}{2}\left(\dot{x}\left(t_{r}\right)\right)^{2} \text { for } t_{r}^{(i)} \in \mathcal{T}_{r}, i \in \mathbb{N}^{*}\right\}
$$

This set of energy is used to estimate the cut-off frequency at each return time $t_{r}^{(i)}$, and is given by $\omega_{c}^{(i)}=$ $N_{c}^{(i)} \omega_{0} \approx E_{r}^{(i)} / \hbar$. The fact that the cut-off frequency can be expressed as a function of the incoming pulse frequency (which is not obvious) is a consequence of an ultrafast (typically femtosecond) process called multiphoton ionization [10]. The recombination times will give us information about when it is a priori necessary to reinitialize the FGA. However, even at some of these times of return, it may be useless to reinitialize the FGA, if the energy of return is not large enough to generate enough high frequency photons. For this reason, we define the set

$$
\mathcal{T}_{r}^{\varepsilon}=\left\{t_{r}^{(i)} \in \mathcal{T}_{r}: E_{r}^{(i)} \geqslant \hbar \varepsilon^{-1}\right\} \subseteq \mathcal{T}_{r} .
$$

Only at times $t$ in $\mathcal{T}_{r}^{\varepsilon}$, which will be very easily numerically estimated, it will be necessary to reinitialize the FGA. In atomic units, which will be used hereafter, $m=1, \hbar=1$ and $e=-1$.

\subsection{Three-step model: numerical experiments}

This section is devoted to some numerical experiments of harmonic generation by intense laser-quantum particle interaction. We consider a $H_{2}^{+}$-molecule with fixed inter-nuclear distance $R_{0}$. The chosen Cauchy data is the ground state (Fig. 1) defined by the eigenfunction of minimal energy

$$
H_{0} \phi_{0}=\lambda_{0} \phi_{0}
$$

where $H_{0}=-\triangle / 2+V$ and $V(x)=-1 /\left|x-R_{0} / 2\right|-1 /\left|x+R_{0} / 2\right|$. The electric field is defined by $E(t)=E_{0} \cos \left(\omega_{0} t\right) e^{-\nu(t-T / 2)^{2}}$, where $T$ is the pulse duration, $\omega_{0}$ its central frequency, $E_{0}$ its amplitude, and $\nu$ a non-negative real parameter. The spatial domain is denoted $(-L, L)$ and the domain is assumed to be large enough to avoid any spurious reflection at its boundary $\pm L$.

The physical parameters in atomic units, are $\nu=4 \times 10^{-5}, E_{0}=0.1$ (corresponding to $\approx 2 \times 10^{14} \mathrm{~W} \cdot \mathrm{cm}^{-2}$ ), $\omega=0.06$ (corresponding to a $800 \mathrm{~nm}$ wavelength pulse) and $T=800$ (corresponding to $\approx 20$ femtoseconds). The internuclear distance is fixed to $R_{0}=2$ and $l=20$. Numerical data are performed using a time step $\Delta t=1 / 30(\approx 0.8$ attosecond $)$ and space step $h=1 / 3(\approx 17$ picometers $)$. We first numerically report in Fig. 2, the electric field (Up), the classical electron position $x(t)$ (middle) and the kinetic energy $m v^{2}(t) / 2$ (Down) by solving Newton's law. The recombination times, corresponding to zeros of $x(t)$, are represented in Fig. 2 (up). As recalled above, harmonics will mainly be generated close to these recombination times. In Fig. 3, we represent at time $T$, the wavefunction (up), the electric field and recombination times (middle), and the temporal harmonics of the dipole moment defined by $d(t)=\int_{\mathbb{R}} x|\psi(t, x)|^{2} d x$, which is denoted by 
$\omega$ the Fourier co-variable associated to $t$, that is $\left(\omega,|\widehat{d}(\omega)|^{2}\right)$ with logscale in the $y$-axis (Down). The latter illustrates the generation of high order harmonics, by electron-nucleus recombination. In order to point out the generation of high frequencies close to recombination times, a wavelet decomposition is also performed. More specifically, in Fig. 4 (up), we show the dipole moment as a function of time, with the recombination times (as classically computed) and in Fig. 4 (Down), we report Haar's wavelet decomposition of the dipole moment, $d(t)$, where the smaller the scale number, the higher the frequency. We observe that the higher the kinetic energy, the higher the generated frequencies. As expected, we observe that new high frequencies are generated close to the recombination times estimated using Corkum's classical model. As a last illustration of HHG in the laser-molecule interaction, we present another test with a more intense external field, in order to enhance high order harmonic generation. The data are the same as above, except that the computational domain is now $(-40,40), I=0.2$ and $N=640$, that is $d x \approx 0.125$. We report in Fig. 5 (Up), the initial state modulus and the wavefunction modulus at final time $T=800$, and the corresponding spatial spectrum (Fourier modes) in logscale (Down).

\section{Two-level FGA-based method}

This section is devoted to the derivation of two two-level methods for multi-frequency TDSE with or without generation of high frequency, from initial time 0 to final time $T>0$. We consider the evolution of the wavefunction, where i) we initially $(t=0)$ separate the Cauchy data into the high spatial frequency contribution from the other spatial frequency contributions (low and moderate), and ii) we independently make evolve in time, these 2 contributions, up to time $T$. The low and moderate frequency contributions will be computed by solving the Schrödinger equation in the quantum regime at $(t, x),(t \in(0, T))$. The high frequency contribution will be computed using FGA at any $(\varepsilon t, \varepsilon x)$. At any time $t$, both contributions are consistently added. The general algorithm that we propose is summarized as follows.

1. Initial decomposition of the Cauchy data, by Fourier filtering into a low/moderate spatial frequency (wavenumber) contribution on a coarse mesh, $\psi_{\mathrm{Q}}$, and a high spatial frequency contribution on a fine mesh. The latter allows for defining the initial FGA, denoted $\psi_{\mathrm{FGA}}$. 
Figure 2: As a function of time, and using classical mechanics. (Up) Electric field and recombination times. (Middle) Electronic position. (Down) Kinetic energy.

2. Time evolution of $\psi_{\mathrm{Q}}$ on the coarse mesh by numerical computation of the TDSE in the quantum regime on the coarse mesh.

3. Time evolution of $\psi_{\mathrm{FGA}}$, approximating the solution of the TDSE in the semi-classical regime.

4. Reconstruction of the global solution on a fine mesh from $\psi_{\mathrm{FGA}}$ and $\psi_{\mathrm{Q}}$.

5. If high frequencies are dynamically generated, the above steps should be repeated. See Section 3.2.

\subsection{Geometry and notations in 1-d}

We denote by $\left\{x_{j}^{h}\right\}_{j \in \mathcal{I}_{x}^{h}}$ (resp. $\left\{x_{j}^{h_{\varepsilon}}\right\}_{j \in \mathcal{I}_{x}^{h_{\varepsilon}}}$ ) the mesh points of a coarse (resp. fine) mesh $\Omega_{h}$ (resp. $\Omega_{h_{\varepsilon}}$ ) of step size $h$ (resp. $h_{\varepsilon}$ ) and where $h_{\varepsilon} \ll h$, with typically $h_{\varepsilon}=h \varepsilon$ and $\varepsilon \ll 1$. The set $\mathcal{I}_{x}^{h} \subseteq \mathbb{Z}$ (resp. $\mathcal{I}_{x}^{h_{\varepsilon}} \subseteq \mathbb{Z}$ ) denotes the index sets on $\Omega_{h}$ (resp. $\Omega_{h_{\varepsilon}}$ ). For $\left\{f_{j}\right\}_{j \in \mathcal{I}_{x}^{h_{\varepsilon}}}$ defined on $\Omega_{h_{\varepsilon}}$ and for any $k \in \mathcal{I}_{x}^{h}$, the projector from $\Omega_{h_{\varepsilon}}$ to $\Omega_{h}$ reads

$$
\left(\mathcal{L}_{h_{\varepsilon} \rightarrow h} f\right)_{k}=\frac{1}{E\left[\varepsilon^{-1}\right]} \sum_{j=1}^{E\left[\varepsilon^{-1}\right]} f_{(k-1) E\left[\varepsilon^{-1}\right]+j},
$$

where $E\left[\varepsilon^{-1}\right]$ denotes the integer part of $\varepsilon^{-1}$. Inversely $\mathcal{L}_{h \rightarrow h_{\varepsilon}}$ is a discrete polynomial interpolation operator from $\Omega_{h}$ to $\Omega_{h_{\varepsilon}}$.

In order to compute the FGA, we have to solve (5) on a $(q, p)$-grid, $D_{q}^{d q} \times \widetilde{D}_{p}^{d p}$ : i) $\widetilde{D}_{p}^{d p}$ with grid points $\left\{p_{k}\right\}_{k \in \mathcal{I}_{p}^{d p}}$ and ii) $D_{q}^{d q}$ with grid points $\left\{q_{k}\right\}_{k \in \mathcal{I}_{q}^{d q}}$, where the corresponding index sets are denoted by $\mathcal{I}_{p}^{d p} \subseteq \mathbb{Z}$ 
Figure 3: At final time. (Up) Wavefunction modulus. (Middle) Electric field and recombination times. (Down) Dipole moment time-harmonics.

and $\mathcal{I}_{q}^{d q} \subseteq \mathbb{Z}$. For $k \in \mathcal{I}_{p}^{d p}$, we have $p_{k}=k d p$ with $d p=2 \pi /|\Omega|$. For the sake of simplicity, we will identify $\left\{q_{j}\right\}_{j \in \mathcal{I}_{q}^{d q}}$ with $\left\{x_{j}^{h}\right\}_{j \in \mathcal{I}_{x}^{h}}$ and $d q=h$, although in practice $D_{q}^{d q}$ may be finer than $\Omega_{h}$, but much coarser for efficiency reason than $\Omega_{h_{\varepsilon}}$, see details in [18].

We denote by $\Delta t$, the constant time step used for solving the TDSE in the quantum regime. Discrete times are denoted $t_{n}=n \Delta t$, with $n \in \mathbb{N}$.

We denote by $\psi^{h}=\left\{\psi_{j}^{h}\right\}_{j \in \mathcal{I}_{x}^{h}}$ (resp. $\psi^{h_{\varepsilon}}=\left\{\psi_{j}^{h}\right\}_{j \in \mathcal{I}_{x}^{h \varepsilon}}$ ) the global discrete solution of (1) at $\left\{x_{j}^{h}\right\}_{j \in \mathcal{I}_{x}^{h}}$ on $\Omega_{h}$ (resp. $\left\{x_{j}^{h_{\varepsilon}}\right\}_{j \in \mathcal{I}_{x}^{h_{\varepsilon}}}$ on $\Omega_{h_{\varepsilon}}$ ), and time $t$, that is

$$
\psi_{j}^{h}(t) \approx \psi\left(t, x_{j}^{h}\right), \quad\left(\operatorname{resp} . \psi_{j}^{h_{\varepsilon}}(t) \approx \psi\left(t, x_{j}^{h_{\varepsilon}}\right)\right) .
$$

We denote by $\psi^{h, n}\left(\right.$ resp. $\left.\psi^{h_{\varepsilon}, n}\right)$ the discrete solution of (1) at $\left\{x_{j}^{h}\right\}_{j \in \mathcal{I}_{x}^{h}} \in \Omega_{h}$ (resp. $\Omega_{h_{\varepsilon}}$ ), at time $t_{n}$, that is

$$
\psi_{j}^{h, n} \approx \psi\left(t_{n}, x_{j}^{h}\right), \quad\left(\operatorname{resp} . \psi_{j}^{h_{\varepsilon}, n} \approx \psi\left(t_{n}, x_{j}^{h_{\varepsilon}}\right)\right)
$$

We denote by $\psi_{\mathrm{FGA}}^{h, n}=\left\{\psi_{F G A, j}^{h, n}\right\}_{j \in \mathcal{I}_{x}^{h}}$ (resp. $\left.\psi_{\mathrm{FGA}}^{h_{\varepsilon}, n}=\left\{\psi_{F G A, j}^{h_{\varepsilon}, n}\right\}_{j \in \mathcal{I}_{x}^{h_{\varepsilon}}}\right)$ the FGA of (1) in the semi-classical limit defined on $\Omega_{h}$ (resp. $\Omega_{h_{\varepsilon}}$ ), including frequencies higher than $\varepsilon^{-1}$. For lower frequencies, we denote by $\psi_{\mathrm{Q}}^{h, n}=\left\{\psi_{Q, j}^{h, n}\right\}_{j \in \mathcal{I}_{x}^{h}}$ (resp. $\psi_{\mathrm{Q}}^{h_{\varepsilon}, n}=\left\{\psi_{Q, j}^{h_{\varepsilon}, n}\right\}_{j \in \mathcal{I}_{x}^{h_{\varepsilon}}}$ ) the solution to (1) in the quantum regime on $\Omega_{h}$ (resp. 
$\Omega_{h_{\varepsilon}}$ ). The global solution $\psi^{h, n}\left(\right.$ resp. $\left.\psi^{h_{\varepsilon}, n}\right)$ on $\Omega_{h}$ (resp. $\Omega_{h_{\varepsilon}}$ ) at time $t_{n}$, satisfies

$$
\psi^{h, n}=\psi_{\mathrm{FGA}}^{h, n}+\psi_{\mathrm{Q}}^{h, n}, \quad\left(\text { resp. } \psi^{h_{\varepsilon, n}}=\psi_{\mathrm{FGA}}^{h_{\varepsilon}, n}+\psi_{\mathrm{Q}}^{h_{\varepsilon}, n}\right) .
$$

\subsection{Two-level algorithm without frequency generation}

We now describe the first algorithm, for a given $\varepsilon \ll 1$, and with $h_{\varepsilon}=\varepsilon h$. For the sake of notation simplicity, we will denote the initial data, $\phi\left(=\phi_{0}\right)$, and we then start from $\phi^{h_{\varepsilon}}:=\psi^{h_{\varepsilon}, 0}=\left\{\phi_{j}^{h_{\varepsilon}}\right\}_{j \in \mathcal{I}_{x}^{h_{\varepsilon}}}$, the Cauchy data projected on the fine mesh $\Omega_{h_{\varepsilon}}$.

Step 1. Initially $\phi^{h_{\varepsilon}}$ is decomposed in i) low/moderate spatial frequency and ii) high spatial frequency contributions. This is easily achieved by Fourier transform, filtering the frequencies below and above $\varepsilon^{-1}$. Notice that FGA is accurate for wavenumbers at most equal to $\varepsilon^{-1}$. We can then introduce a free parameter $\eta \gtrsim \varepsilon$, and construct a FGA to approximate the contribution of frequencies higher than $\eta^{-1}\left(\lesssim \varepsilon^{-1}\right)$ rather than $\varepsilon^{-1}$ in order to give more flexibility to the algorithm. Lower frequency contribution will be computed on $\Omega_{h}$. With $p_{k}=k d p$, we then define:

$$
v_{\eta, k}^{h_{\varepsilon}}= \begin{cases}h_{\varepsilon} \sum_{j \in \mathcal{I}_{x}^{h_{\varepsilon}}} e^{-i p_{k} \cdot x_{j}^{h_{\varepsilon}}} \phi_{Q, j}^{h_{\varepsilon}}, & \text { for }\left|p_{k}\right| \leqslant \eta^{-1}, \\ 0 & \text { for }\left|p_{k}\right|>\eta^{-1} .\end{cases}
$$

Next, we define for $j \in \mathcal{I}_{x}^{h_{\varepsilon}}$ (omitting the index $\eta$ in the LHS to lighten the notations)

$$
\phi_{Q, j}^{h_{\varepsilon}}=\frac{d p}{(2 \pi)^{d}} \sum_{k \in \mathcal{I}_{p}^{d p}} e^{i p_{k} \cdot x_{j}^{h_{\varepsilon}}} v_{\eta, k}^{h_{\varepsilon}},
$$

and, we project this function onto the coarse mesh $\Omega_{h}$

$$
\phi_{\mathrm{Q}}^{h}=\mathcal{L}_{h_{\varepsilon} \rightarrow h} \phi_{\mathrm{Q}}^{h_{\varepsilon}} .
$$



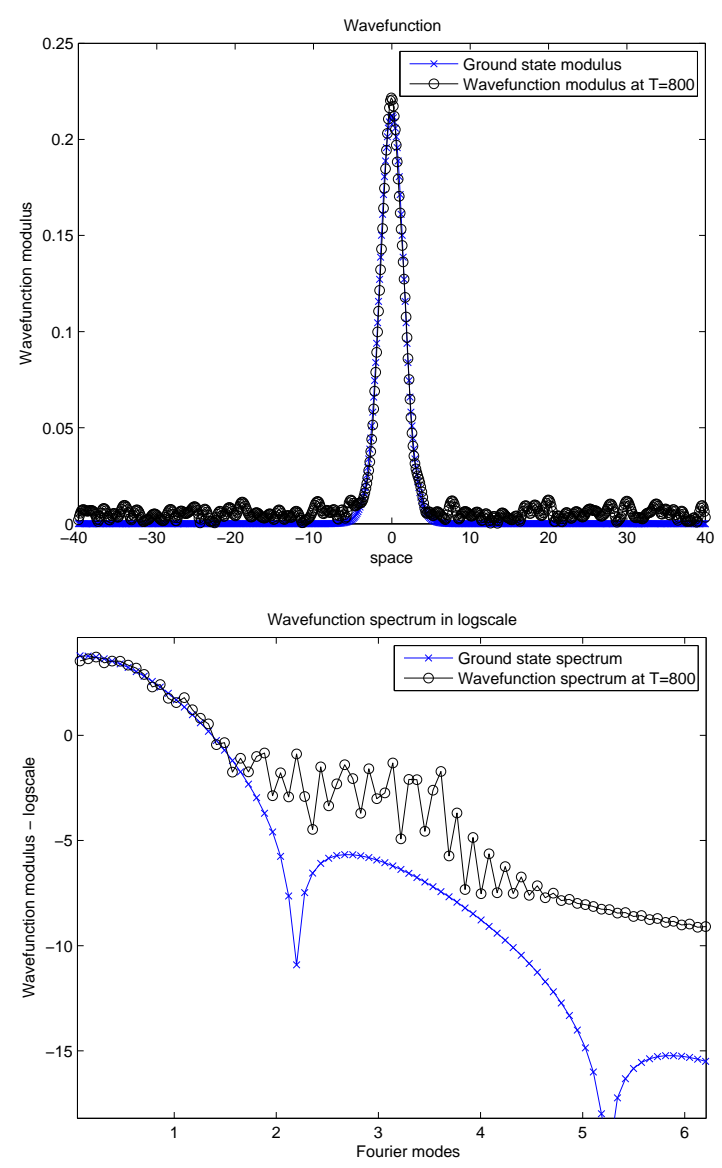

Figure 5: (Up) Initial wavefunction modulus and wavefunction modulus at time $T=800$. (Down) Corresponding spatial Fourier modes.

This gives us the Cauchy data for computing the low/moderate frequency contribution in the quantum regime. We next define the initial FGA contribution.

Step 1. According to [18], we define the FGA contribution at $x_{j}^{h_{\varepsilon}}$ in $\Omega_{h_{\varepsilon}}$, as

$$
\begin{aligned}
\phi_{F G A, j}^{h_{\varepsilon}}= & \frac{1}{(2 \pi \varepsilon)^{3 / 2}} \sum_{(k, l) \times \mathcal{I}_{q}^{h} \times \mathcal{I}_{p}^{h}} a\left(0, q_{k}, p_{l}\right) w_{\eta}^{h_{\varepsilon}}\left(q_{k}, p_{l}\right) \\
& \times e^{\frac{i}{\varepsilon}\left(S\left(0, q_{k}, p_{l}\right)+P\left(0, q_{k}, p_{l}\right) \cdot\left(x_{j}^{h_{\varepsilon}}-Q\left(0, q_{k}, p_{l}\right)\right)\right)} e^{-\frac{1}{2 \varepsilon}\left|x_{j}^{h_{\varepsilon}}-Q\left(0, q_{k}, p_{l}\right)\right|^{2}} d p d q,
\end{aligned}
$$

with

$$
w_{\eta}^{h_{\varepsilon}}\left(q_{k}, p_{l}\right)=h_{\varepsilon} \sum_{j \in \mathcal{I}_{x}^{h_{\varepsilon}}} e^{-\frac{i}{\varepsilon} p_{l} \cdot\left(x_{j}^{h_{\varepsilon}}-q_{k}\right)-\frac{1}{2 \varepsilon}\left|x_{j}^{h_{\varepsilon}}-q_{k}\right|^{2}}\left(\phi_{j}^{h_{\varepsilon}}-\phi_{Q, j}^{h_{\varepsilon}}\right),
$$

and where we have chosen $q_{j}=x_{j}^{h}$ and $d q=h$. In order to determine $a$ and $S$ in the above expression, it is necessary to rewrite the initial data $\phi_{0}$ as follows:

$$
\phi_{0}(x)=A(x) \exp (i S(x)) .
$$

Setting $x^{\prime}=\varepsilon x$, the coordinates in which FGA are usually expressed, we have

$$
\phi_{0}\left(x^{\prime}\right)=A\left(x^{\prime} / \varepsilon\right) \exp \left(i S\left(x^{\prime} / \varepsilon\right)\right)=A\left(x^{\prime} / \varepsilon\right) \exp \left(i \varepsilon S\left(x^{\prime} / \varepsilon\right) / \varepsilon\right) .
$$


From Section 1.2, we easily deduce $S$ and $a$ on $\Omega_{h}$. Thus, we have defined $\phi_{\mathrm{FGA}}^{h_{\varepsilon}}=\left\{\phi_{F G A, j}^{h_{\varepsilon}}\right\}_{j \in \mathcal{I}_{x}^{h_{\varepsilon}}}$. Moreover, by construction we have:

$$
\phi^{h_{\varepsilon}}=\phi_{\mathrm{Q}}^{h_{\varepsilon}}+\phi_{\mathrm{FGA}}^{h_{\varepsilon}} .
$$

Step 2. In order to make evolve the global wavefunction in time, we need to consistently update the low/moderate frequency contribution to the high frequency one. As we have assumed that there is no high frequency generation during the time evolution, and setting $t^{\prime}=\varepsilon t$

$$
\begin{aligned}
\psi_{F G A, j}^{h_{\varepsilon}}\left(t^{\prime}\right)= & \frac{1}{(2 \pi \varepsilon)^{3 / 2}} \sum_{(k, l) \in \mathcal{I}_{q}^{h} \times \mathcal{I}_{p}^{h}} a\left(t^{\prime}, q_{k}, p_{l}\right) w_{\eta}^{h_{\varepsilon}}\left(q_{k}, p_{l}\right) \\
& \times e^{\frac{i}{\varepsilon}\left(S\left(t^{\prime}, q_{k}, p_{l}\right)+P\left(t^{\prime}, q_{k}, p_{l}\right) \cdot\left(x_{j}^{h_{\varepsilon}}-Q\left(t^{\prime}, q_{k}, p_{l}\right)\right)\right)} e^{-\frac{1}{2 \varepsilon}\left|x_{j}^{h_{\varepsilon}}-Q\left(t^{\prime}, q_{k}, p_{l}\right)\right|^{2}} d p d q,
\end{aligned}
$$

which is an approximate solution to

$$
\mathrm{i} \varepsilon \frac{\partial \psi^{\varepsilon}}{\partial t^{\prime}}\left(t^{\prime}, x^{\prime}\right)=-\frac{\varepsilon^{2}}{2} \triangle \psi^{\varepsilon}\left(t^{\prime}, x^{\prime}\right)+V^{\varepsilon}\left(t^{\prime}, x^{\prime}\right) \psi^{\varepsilon}\left(t^{\prime}, x^{\prime}\right)
$$

with the Cauchy data (13). This is numerically performed by solving (5) on $D_{q}^{d q} \times \widetilde{D}_{p}^{d p}$, with $d q=h$. Note that as there is no frequency generation, this reconstruction can be performed only at final time. Regarding low/moderate frequencies, $\phi_{\mathrm{Q}}^{h}$ is computed at any time $t \leqslant T$, by solving

$$
\mathrm{i} \frac{\partial \psi}{\partial t}(t, x)=-\frac{1}{2} \triangle \psi(t, x)+V(t, x) \psi(t, x),
$$

with the Cauchy data defined in (12). It is important to recall that $\psi_{\mathrm{Q}}^{h}(t)$ and $\psi_{\mathrm{FGA}}^{h_{\varepsilon}}(\varepsilon t)$ are computed at different positions and times. At any $t \leqslant T$, we then denote by $\tilde{t}:=t / \varepsilon$, and we have

$$
\psi^{h_{\varepsilon}}(t)=\mathcal{L}_{h \rightarrow h_{\varepsilon}} \psi_{\mathrm{Q}}^{h}(t)+\psi_{\mathrm{FGA}}^{h_{\varepsilon}}(\varepsilon \tilde{t}), \psi^{h}(t)=\psi_{\mathrm{Q}}^{h}(t)+\mathcal{L}_{h_{\varepsilon} \rightarrow h} \psi_{\mathrm{FGA}}^{h_{\varepsilon}}(\varepsilon \tilde{t}) .
$$

At the discrete level in time, for $t_{n}=n \Delta t$, and $t_{\varepsilon, n}=n \Delta t / \varepsilon$ with $k \in \mathbb{N}^{*}$,

$$
\psi^{h_{\varepsilon}, n}=\mathcal{L}_{h \rightarrow h_{\varepsilon}} \psi_{\mathrm{Q}}^{h, n}+\psi_{\mathrm{FGA}}^{h_{\varepsilon}, n}, \psi^{h, n}=\psi_{\mathrm{Q}}^{h, n}+\mathcal{L}_{h_{\varepsilon} \rightarrow h} \psi_{\mathrm{FGA}}^{h_{\varepsilon}, n} .
$$

\subsection{Two-level scheme with frequency generation}

In this subsection, we use similar notations as in Section 3.2, and we assume that harmonics, including high harmonics, are generated by recombination during the field-particle interaction. As a consequence, and unlike Section 3.2, FGA can not be computed from $\phi_{0}$ only, but additional reinitializations are necessary. It is also important to notice that unlike the previous case (no harmonic generation), we intend to use this two-level approach, only when very high frequencies are generated. The latter will be computed using a FGA and then should be seen as an improvement of standard solvers for TDSE in the fully quantum regime. All the other frequencies, from low to high will be treated by a standard real space method. We now introduce a small parameter $1 \gg \delta>\varepsilon>0$, and we recall that $\left\{t_{r}^{(k)}\right\}_{k} \in \mathcal{T}_{r}^{\varepsilon}$ denote the recombination times (10).

Step 0. The very first step consists of the estimation of the recombination times and the corresponding return energies allowing to determine $\mathcal{T}_{r}^{\varepsilon}$. In the following indices $n_{r}^{(i)}$ denote the integer part of $t_{r}^{(i)} / \Delta t$, where $t_{r}^{(i)} \in \mathcal{T}_{r}^{\varepsilon}$. We also denote by $\Delta n$ the integer part of $\delta / \Delta t$. This can very rapidly and efficiently be performed by a RK4 scheme.

Step 1. By definition, for all $n \in\left(0, n^{(1)}-\delta\right)$, there is no high frequency generation. As a consequence, the strategy presented in Section 3.2, is applicable in this time interval. That is, at any $n \leqslant n_{r}^{(1)}-\Delta n$, we perform Steps 1. and 2. from Section 3.2, and we determine: 
- $\psi_{\mathrm{Q}}^{h}$ on $\Omega_{h}$ up to time $t_{r}^{(1)}-\delta$, by solving (1) in the quantum regime that is: $\psi_{\mathrm{Q}}^{h, n_{r}^{(1)}-\Delta n} \approx \psi_{\mathrm{Q}}^{h}\left(t_{r}^{(1)}-\delta\right)$.

- $\psi_{\mathrm{FGA}}^{h_{\varepsilon}}$ using Section 1.2, and such that $\psi_{\mathrm{FGA}}^{h_{\varepsilon}, n_{r}^{(1)}-\Delta n} \approx \psi_{\mathrm{FGA}}^{h_{\varepsilon}}\left(t_{r}^{(1)}-\delta\right)$.

- $\psi^{h_{\varepsilon}, n_{r}^{(1)}-\Delta n}$ is then constructed from $\psi_{\mathrm{Q}}^{h, n_{r}^{(1)}-\Delta n}$ and $\psi_{\mathrm{FGA}}^{h_{\varepsilon}, n_{r}^{(1)}-\Delta n}$.

Step 2. If $t_{r}^{(1)} \in \mathcal{T}_{r}^{\varepsilon}$, harmonics higher than $\eta^{-1} \lesssim \varepsilon^{-1}$ are generated in the time interval $\left(t_{r}^{(1)}-\delta, t_{r}^{(1)}+\delta\right)$, then for $t \in\left(t_{r}^{(1)}-\delta, t_{r}^{(1)}+\delta\right)$, we construct $\psi^{h_{\varepsilon}}(t)$ by solving $(1)$ on $\Omega_{h_{\varepsilon}}$ with Cauchy data $\psi^{h_{\varepsilon}}\left(t_{r}^{(1)}-\delta\right)$. The computation on the fine mesh, but on a very short time interval $(2 \delta)$, of the quantum Schrödinger equation will allow for an accurate numerical generation of high harmonics. Then, for $t \in\left(t_{r}^{(1)}+\delta, t_{r}^{(2)}-\delta\right)$, high frequency generation is not expected, and the FGA can then be used on this new time interval, and we apply the same the strategy as Step 1 from $t=t_{r}^{(1)}+\delta$ to $t_{r}^{(2)}-\delta$. At the discrete level, we proceed as follows.

- From Step 1, we get: $\psi_{\mathrm{Q}}^{h, n_{r}^{(1)}-\Delta n}$ and $\psi_{\mathrm{FGA}}^{h_{\varepsilon}, n_{r}^{(1)}-\Delta n}$. We interpolate $\psi_{\mathrm{Q}}^{h, n_{r}^{(1)}-\Delta n}$ on $\Omega_{h_{\varepsilon}}$, and then reconstruct the wavefunction on the fine mesh $\Omega_{h_{\varepsilon}}$

$$
\psi^{h_{\varepsilon}, n_{r}^{(1)}-\Delta n}=\mathcal{L}_{h \rightarrow h_{\varepsilon}} \psi_{\mathrm{Q}}^{h, n_{r}^{(1)}-\Delta n}+\psi_{\mathrm{FGA}}^{h_{\varepsilon}, n_{r}^{(1)}-\Delta n}
$$

- We compute $\psi^{h_{\varepsilon}, n}$, for $n$ such that $n_{r}^{(1)}-\Delta n \leqslant n \leqslant n_{r}^{(1)}+\Delta n$, by solving (1) on $\Omega_{h_{\varepsilon}}$ by finite differences.

- From $\psi^{h_{\varepsilon}, n_{r}^{(1)}+\Delta n}$, we then construct: i) the quantum contribution $\psi_{\mathrm{Q}}^{h, n_{r}^{(1)}+\Delta n}$ using the projection operator $\mathcal{L}_{h_{\varepsilon} \rightarrow h}$, and $\psi_{\mathrm{FGA}}^{h_{\varepsilon}, n_{r}^{(1)}+\Delta n}$ using the same strategy as Step 1 .

- Evolution of $\psi_{\mathrm{Q}}^{h}$ and $\psi_{\mathrm{FGA}}^{h_{\varepsilon}}$ follow Step 2. of Section 3.2, for computing $\psi^{h_{\varepsilon}, n}$ and $\psi_{\mathrm{FGA}}^{h_{\varepsilon}, n}$ for $n$ such that $n_{r}^{(1)}+\Delta n \leqslant n \leqslant n_{r}^{(2)}-\Delta n$.

Step 3. Now for any $t_{r}^{(i)} \in \mathcal{T}_{r}^{\varepsilon}$, with $i>1$, the principle is similar as above:

- Reconstruction of the global wavefunction $\psi^{h_{\varepsilon}, n_{r}^{(i)}-\Delta n}$ on $\Omega_{h_{\varepsilon}}$.

- Evolution of $\psi^{h_{\varepsilon}, n}$, by solving (1) on $\Omega_{h_{\varepsilon}}$ by finite differences, for $n$ such that $n_{r}^{(i)}-\Delta n \leqslant n \leqslant n_{r}^{(i)}+\Delta n$.

- Reconstruction of $\psi_{\mathrm{Q}}^{h, n_{r}^{(i)}+\Delta n}$ and $\psi_{\mathrm{FGA}}^{h, n_{r}^{(i)}+\Delta n}$ from $\psi^{h_{\varepsilon}, n_{r}^{(i)}+\Delta n}$ and evolution as in Step 2.

Although this two-level method requires interpolation and computation at the fine level $\left(\Omega_{h_{\varepsilon}}\right)$, the reinitialization of the FGA is only necessary at times $t_{r}^{(i)} \in \mathcal{T}_{r}^{\varepsilon}[10]$.

\subsection{Theoretical estimation of the computational complexity}

Let us denote by $N_{T}$, the number of time iterations to reach the final time $T$. In $d$ dimension, we denote by $N_{f}^{d}$ (resp. $N_{c}^{d}$ ) the number of mesh points in the fine (resp. coarse) mesh. We have $N_{c} / N_{f} \approx \varepsilon$. We denote by $N_{p}^{d}$ the number of grid points in $\widetilde{D}_{p}^{d p}$, and $N_{q}^{d}$ the number of grid points in $D_{q}^{d q}$, see Section 1.2. Typically, $N_{q} \approx N_{c}$, and $N_{p} / N_{q} \ll 1$ for localized high frequencies [18]. The overall computational complexity, $C_{\mathrm{f}}$, to compute the solution to (1) with a Crank-Nicolson difference scheme on the fine mesh, is given by

$$
C_{\mathrm{f}}=\mathcal{O}\left(N_{T}\left(N_{\mathrm{f}}^{d}\right)^{\alpha}\right),
$$

with $3 \gtrsim \alpha>1$ related to the computational complexity to solve sparse linear systems.

Now, the overall two-level method with recombination respectively requires: 
- $C_{\mathrm{c}}^{(1)}=\mathcal{O}\left(N_{T}\left(N_{c}^{d}\right)^{\alpha}\right)$ operations in order to compute the low/moderate frequency contribution on the coarse mesh, by solving (1) by finite differences.

- $C_{\mathrm{c}}^{(2)}=\mathcal{O}\left(N_{T}\left(N_{q}^{d} N_{p}^{d}\right)\right)$ operations to compute (5) up to final time $T$.

- Let us denote by $R_{r}^{\varepsilon}=\# \mathcal{T}_{r}^{\varepsilon}$ the number of recombination times necessitating a reinitialization of the FGA. Then, the Fourier filtering, window Fourier transforms, projections and interpolations require a total of $C_{\mathrm{c}}^{(3)}=\mathcal{O}\left(R_{r}^{\varepsilon} N_{\mathrm{f}}^{d} \log N_{\mathrm{f}}^{d}\right)$ operations.

- Finally, in order to properly generate the high order harmonics, it is necessary to solve the TDSE by finite differences on the fine mesh, during $2 \Delta n$ iterations, with $\Delta n \ll n$. The corresponding computational complexity is given by

$$
C_{\mathrm{c}}^{(4)}=\mathcal{O}\left(R_{r}^{\varepsilon} \Delta n\left(N_{\mathrm{f}}^{d}\right)^{\alpha}\right) .
$$

The overall computational complexity for the two-level method with recombination, $C_{\mathrm{c}}^{r}$, is then

$$
\left.C_{\mathrm{c}}^{r}=\sum_{i=1}^{4} C_{\mathrm{c}}^{(i)}=\mathcal{O}\left(R_{r}^{\varepsilon} \Delta n\left(N_{\mathrm{f}}^{d}\right)^{\alpha}\right)+\mathcal{O}\left(R_{r}^{\varepsilon} N_{\mathrm{f}}^{d} \log N_{\mathrm{f}}^{d}\right)+\mathcal{O}\left(N_{T}\left(N_{c}^{d}\right)^{\alpha}+N_{q}^{d} N_{p}^{d}\right)\right) .
$$

We also deduce that the overall computational complexity for the two-level method without recombination, $C_{\mathrm{c}}^{o}$, is given by

$$
\left.C_{\mathrm{c}}^{o}=\mathcal{O}\left(N_{\mathrm{f}}^{d} \log N_{\mathrm{f}}^{d}\right)+\mathcal{O}\left(N_{T}\left(N_{c}^{d}\right)^{\alpha}+N_{q}^{d} N_{p}^{d}\right)\right) .
$$

From a practical point of view, for $\varepsilon \ll 1$, we have

$$
N_{c} \ll N_{\mathrm{f}}, N_{T} / R_{r}^{\varepsilon} \Delta n \gg 1
$$

We then expect: $C_{\mathrm{c}}^{o} \leqslant C_{\mathrm{c}}^{r} \ll C_{\mathrm{f}}$.

\section{Numerical Simulations}

Some numerical illustrations of the proposed two-level techniques are presented in this section.

\subsection{Two-level method: initial decomposition}

The first test is dedicated to the decomposition of the Cauchy data into high and low/moderate spatial frequency (wavenumber) contributions and its reconstruction from i) a FGA and ii) the projected Cauchy data on a coarse mesh. We consider a Cauchy data, $\phi=\phi_{0}$ constituted by 3 (low, moderate, high) spatial frequencies, respectively denoted by $\nu_{i}, i=1,2,3$. We also denote $\varepsilon_{i}=1 / 2 \pi \nu_{i}$, and $A_{i}$ the amplitudes such that

$$
\phi_{0}(x)=f(x) \sum_{j=1}^{3} A_{j} e^{i x / \varepsilon_{j}} .
$$

In (16), $f$ is an envelope function, which is a Gaussian function centered at 0 . We denote by $\phi^{L}$ the low/moderate frequency component of the Cauchy data, and by $\phi^{H}$ its high frequency component. That is

$$
\phi^{L}(x)=f(x) \sum_{j=1}^{2} A_{j} e^{i x / \varepsilon_{j}}, \quad \phi^{H}(x)=f(x) A_{3} e^{i x / \varepsilon_{3}} .
$$

In order to initiate the two-level algorithm, a coarse finite difference grid is introduced which only allows for the accurate representation of the spatial frequencies $\nu_{1}$ and $\nu_{2}$ (not $\left.\nu_{3}\right)$. The high frequency contribution is 
reconstructed by FGA in the semi-classical regime. In order to estimate the numerical accuracy of the twolevel reconstruction, we compare the reconstructed Cauchy data, $\phi_{\mathrm{FGA}}^{h_{\varepsilon}}+\mathcal{L}_{h \rightarrow h_{\varepsilon}} \phi_{\mathrm{Q}}^{H}$, with the exact Cauchy data (16) projected on the fine finite difference grid $\Omega_{h_{\varepsilon}}$, that is $\phi^{h_{\varepsilon}}$. The parameters are given by $c=-5$, $A_{i}=1$ with $i=1,2,3, \nu_{1}=5, \nu_{2}=25, \nu_{3}=50$ and $\varepsilon_{1} \approx 3.2 \times 10^{-2}, \varepsilon_{2} \approx 6.4 \times 10^{-3}$ and $\varepsilon_{3} \approx 3.2 \times 10^{-3}$. The domain is given by: $(-1,1)$ and the envelope function is $f(x)=e^{-10 x^{2}}$. The numerical parameters are as follows: $h_{\varepsilon}=2.5 \times 10^{-3}$ and $h=10^{-2}, N=600$. In Fig. 6 , we report the Fourier modes of the Cauchy data $\phi_{0}$. The first two peaks are referred as the low and moderate frequencies (wavenumbers), and the last one to the high frequency. We report in Fig. 7 (left), the real part of the exact Cauchy data projected on

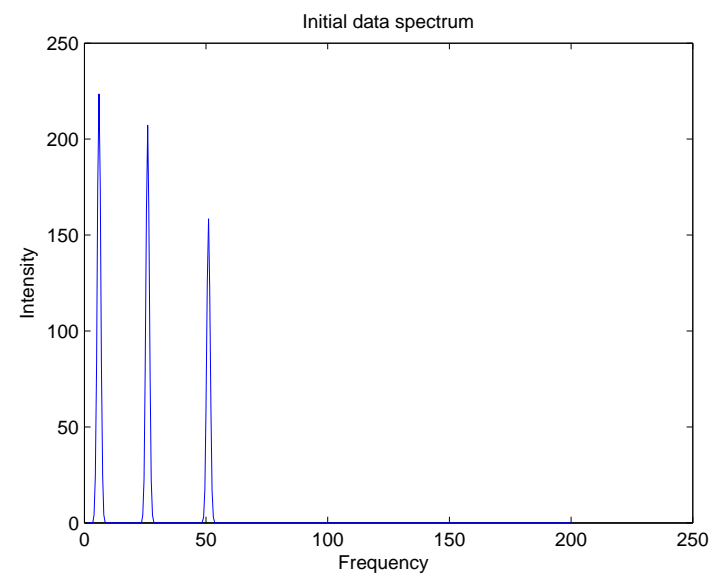

Figure 6: Cauchy data spectrum.

the fine mesh Real $\left(\phi_{\text {ref }}^{h_{\varepsilon}}\right)$, and of the reconstructed Cauchy data Real $\left(\phi_{\mathrm{Q}}^{h_{\varepsilon_{3}}}+\phi_{\mathrm{FGA}}^{h_{\varepsilon_{3}}}\right)$ corresponding the sum of the semi-classical (high frequency) and quantum (low/moderate) contributions. The pointwise error real part between the reconstructed wavefunction and the wavefunction of reference on the fine mesh $\phi_{\mathrm{ref}}^{h_{\varepsilon}}$, that is $\operatorname{Real}\left(\phi_{\mathrm{ref}}^{h_{\varepsilon}}-\left(\phi_{\mathrm{Q}}^{h_{\varepsilon_{3}}}+\phi_{\mathrm{FGA}}^{h_{\varepsilon_{3}}}\right)\right)$, is also reported in Fig. 7 (right). As expected a very good accuracy is obtained.
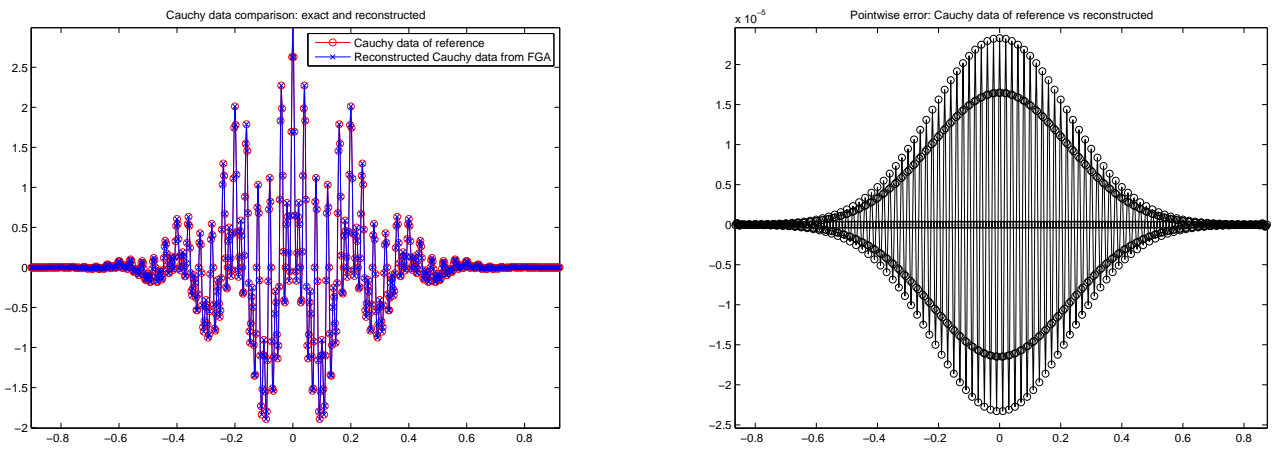

Figure 7: Comparison between the Cauchy data of reference on the fine mesh, with the reconstructed Cauchy data from FGA and the Cauchy data on the coarse mesh: (Left) Real part. (Right) Pointwise error. 


\subsection{Two-level method without frequency generation}

We now consider the time dependent equation (1) without harmonic generation nor external field, see Section 3.2. This constitutes the most simple situation, as the initial decomposition is sufficient to separate at any time, the high and low/moderate frequency contributions.

Test 1 . The first test we consider is as follows. The potential $V$ is defined by

$$
V(x)=-\exp \left(0.2 x^{2}\right) .
$$

The computational domain is $(-64,64)$ and the final computational time is given by $T=10$. The domain is assumed large enough to avoid any spurious reflections. We assume that the Cauchy data possesses again low, moderate and high spatial frequencies. More specifically, we take:

$$
\phi_{0}(x)=\exp \left(-10 \varepsilon^{2} x^{2}\right)\left(\exp \left(i\left(x+\varepsilon x^{2}\right)\right)+4 \exp \left(i\left(\varepsilon^{3 / 4} x+\varepsilon^{7 / 4} x^{2}\right)\right)+3 \exp \left(i\left(\varepsilon x+\varepsilon^{2} x^{2}\right)\right)\right),
$$

with $\varepsilon=2^{-5}$. Numerical data are as follows. We take $h=1.28, h_{\varepsilon}=\varepsilon h=0.04, \Delta t \approx 0.067$ corresponding to 150 iterations. The Fourier filtering has been applied at $\eta^{-1}=4 \varepsilon^{-1} / 5 \approx 25.6$. That is, the low/moderate frequency contribution is computed on the coarse mesh for frequencies less than $\eta^{-1}$ (11), by FGA for frequencies higher than $\eta^{-1}$, see Step 1. of Section 3.2 for details. The Cauchy data is represented in Fig. 8. More specifically, we report the real and imaginary part of the wavefunction $\phi_{\mathrm{Q}}^{h_{\varepsilon}}$ on the fine mesh $\left(\Omega_{h_{\varepsilon}}\right)$, $\phi_{\mathrm{Q}}^{h}$ on the coarse mesh $\left(\Omega_{h}\right)$, the filtered FGA, and $\phi_{\mathrm{FGA}}^{h_{\varepsilon}}+\mathcal{L}_{h \rightarrow h_{\varepsilon}} \phi_{\mathrm{Q}}^{h}$ the overall reconstructed initial data. The modulus of the initial pointwise error between the reconstructed wavefunction and the wavefunction of reference on the fine mesh $\phi_{\text {ref }}^{h_{\varepsilon}},\left|\phi_{\mathrm{FGA}}^{h_{\varepsilon}}+\mathcal{L}_{h \rightarrow h_{\varepsilon}} \phi_{\mathrm{Q}}^{h}-\phi_{\mathrm{ref}}^{h_{\varepsilon}}\right|$, is represented in Fig. 9. At time $T=10$, we then report the real and imaginary part of the wavefunction $\psi_{\mathrm{Q}}^{h_{\varepsilon}}(T)$ on the fine mesh $\left(\Omega_{h_{\varepsilon}}\right), \psi_{\mathrm{Q}}^{h}(T)$ on the coarse mesh $\left(\Omega_{h}\right)$, the filtered FGA, and $\psi_{\mathrm{FGA}}^{h_{\varepsilon}}(T)+\mathcal{L}_{h \rightarrow h_{\varepsilon}} \psi_{\mathrm{Q}}^{h}(T)$ the overall reconstructed wavefunction on the fine mesh. The modulus of the final pointwise error between the reconstructed wavefunction and the wavefunction of reference computed on the fine mesh $\psi_{\text {ref }}^{h_{\varepsilon}}(T),\left|\psi_{\mathrm{FGA}}^{h_{\varepsilon}}(T)+\mathcal{L}_{h \rightarrow h_{\varepsilon}} \psi_{\mathrm{Q}}^{h}(T)-\psi_{\mathrm{ref}}^{h_{\varepsilon}}(T)\right|$, is represented in Fig. 11. The results show a very good accuracy of the two-level solution. We notice in particular, that between times 0 and $T$, the sup-norm in space of the error is almost constant:

$$
\left\|\phi_{\mathrm{FGA}}^{h_{\varepsilon}}+\mathcal{L}_{h \rightarrow h_{\varepsilon}} \phi_{\mathrm{Q}}^{h}-\phi_{\mathrm{ref}}^{h_{\varepsilon}}\right\|_{\infty} \approx\left\|\psi_{\mathrm{FGA}}^{h_{\varepsilon}}(T)+\mathcal{L}_{h \rightarrow h_{\varepsilon}} \psi_{\mathrm{Q}}^{h}(T)-\psi_{\mathrm{ref}}^{h_{\varepsilon}}(T)\right\|_{\infty} \approx 7 \times 10^{-4} .
$$

Test 2. The following experiment is an introductory test for external excitation by a laser field. We assume that a two-frequency wavepacket is subject in vacuum $(V=0)$ to an external field.

$$
\mathrm{i} \frac{\partial \psi}{\partial t}(t, x)=-\frac{1}{2} \triangle \psi(t, x)+x E(t) \psi(t, x) .
$$

The external field is given by $E(t)=I \sin (\omega t) \exp \left(-\nu(t-T / 2)^{2}\right)$, where in atomic units, the pulse duration is $T=16$, its central frequency is $\omega=1$, its intensity is $I=0.2$, and the parameter $\nu$ is fixed to $5 \times 10^{-3}$. The initial wavefunction is given by

$$
\phi_{0}(x)=\exp \left(-10 \varepsilon^{2} x^{2}\right)(\exp (\mathbf{i} x)+2 \exp (\mathbf{i} \varepsilon x))
$$

with $\varepsilon=2^{-5}$. The computational domain is $(-64,64)$. The numerical data are as follows. We take $\eta^{-1}=$ $4 / 5 \varepsilon^{-1}=25.6, h_{\varepsilon}=0.04, h=0.32$, and $\Delta t=0.032$. We first represent in Fig. 12, the pulse as a function of time. In Fig. 13, we report the real and imaginary parts of the Cauchy data of reference (projection on the fine mesh), as well as the reconstruction one from the FGA and the coarse mesh wavefunction. At final time $T=16$, we report in Fig. 14 the wavefunction of reference computed on a fine mesh, and the reconstructed wavefunction. We also represent in Fig. 15, at initial and final times, the spatial spectrum of the wavefunction of reference and of the reconstructed one. This last graph shows that, as expected, no frequency were generated. As a consequence, the method presented in Section 3.2 is perfectly adapted to this type of problems. 
Figure 8: Comparison at time $t=0$ between i) the initial wavefunction on the fine mesh, ii) the reconstructed wavefunction on the fine mesh from the FGA and the wavefunction on the coarse mesh, iii) the wavefunction on the coarse mesh, and iv) the FGA contribution. (Up) Real part. (Down) Imaginary part.

\subsection{Two-level method with frequency generation: preliminary tests}

In this section, we propose some preliminary numerical experiments illustrating the strategy presented in Section 3.3, for multiscale TDSE with harmonic generation. A forthcoming paper will be dedicated to more detailed and realistic benchmarks, as well as the optimization of the two-level algorithm with generation of high frequencies. One of the key points of the algorithm from Section 3.3 is the computation of the recombination times, when the generation of new high frequencies occurs. As shown in Section 2.2, it is possible to approximately determine the recombination times in order to determine when we need to 
Figure 9: Pointwise error between the initial wavefunction on the fine mesh, and the reconstructed wavefunction on the fine mesh.

reinitialize the FGA. Notice that as the Cauchy data is chosen as a low energy eigenfunction, the electronic wavefunction initially contains usually only low intensity high spatial frequencies. From a practical point of view, we proceed as follows.

- Wavefunction initialization and time evolution on the coarse mesh, until recombination times.

- Construction of the wavefunction on the fine mesh prior to the harmonic generation (HHG).

- Evolution on the fine mesh during the HHG.

- Construction and evolution of i) the FGA for the high frequency contribution and ii) of the wavefunction contribution for low/moderate frequency contribution on the coarse mesh.

We consider the following TDSE for 1 electron and 2 fixed nuclei,

$$
i \frac{\partial \psi}{\partial t}(t, x)=H_{0} \psi(t, x)+x E(t) \psi(t, x),
$$

where $H_{0}=-\frac{1}{2} \triangle+V(x)$ with

$$
V(x)=-10 \exp \left(-10(x-R / 2)^{2}\right)-10 \exp \left(-10(x+R / 2)^{2}\right) .
$$

The initial state $\phi_{0}$ is the ground state of $H_{0}$, that is $H_{0} \phi_{0}=\lambda_{0} \phi_{0}$, where $\lambda_{0}$ is the smallest eigenvalue of $H_{0}$. $R$ denotes the fixed internuclear distance between the two nuclei, which is here given by $R=15 / 2^{5}$. The laser field is given by $E(t)=I \sin (\omega t) \exp \left(-\nu(t-T / 2)^{2}\right)$, with $t \in(0, T)$ and $T=32, I=15 / 2^{5}$, $\nu=4 \times 10^{-2}$. In the following we take $\varepsilon=2^{-5}$ and the computational domain is given $\left(-3 \varepsilon^{-1} / 2,3 \varepsilon^{-1} / 2\right)$. The numerical data are: $h_{\varepsilon}=4.69 \times 10^{-2}, h=32 h_{\varepsilon}=1.5, \Delta t=8 \times 10^{-3}$. We fix $d p=8 / \varepsilon$ and $d q=\Delta x$ and the threshold is $\eta^{-1}=5 \varepsilon^{-1} / 4$. The frequency contribution below $\eta^{-1}$ is computed by finite differences (7), and above $\eta^{-1}$ by FGA. In Fig. 16, we report the modulus of i) the wavefunction of reference $\phi_{\text {ref }}^{h_{\varepsilon}}$, 
Figure 10: Comparison at time $T=10$ between i) the wavefunction of reference on the fine mesh, ii) the reconstructed wavefunction on the fine mesh from the FGA and the wavefunction on the coarse mesh, iii) the wavefunction on the coarse mesh, and iv) the FGA contribution. (Up) Real part. (Down) Imaginary part.

which is the projection on the fine mesh of the Cauchy data $\phi_{0}$, ii) $\phi_{\mathrm{Q}}^{h}$ the coarse mesh projection of $\phi_{0}$, iii) $\phi^{h_{\varepsilon}}$ the reconstructed Cauchy data from the FGA and $\phi_{\mathrm{Q}}^{h}$, and finally iv) $\phi_{\mathrm{FGA}}$, the FGA corresponding to the high frequency contribution. The corresponding spatial frequencies of the Cauchy data (groundstate), reconstructed Cauchy data, and the Cauchy data of reference are reported in Fig. 17. We compare in Fig. 18 the (high) frequency contribution above $\eta^{-1}$ of the groundstate of reference with $\phi_{\mathrm{FGA}}^{h_{\varepsilon}}$. We report in Fig. 19 , the laser field with corresponding approximate recombination times, that is when the FGA needs to be reinitialized (Up), the electron position (middle) and the return energy (Down). Similarly we represent at 
Figure 11: Pointwise error between i) the wavefunction of reference on the fine mesh, and ii) the reconstructed wavefunction on the fine mesh, at time $T=10$.

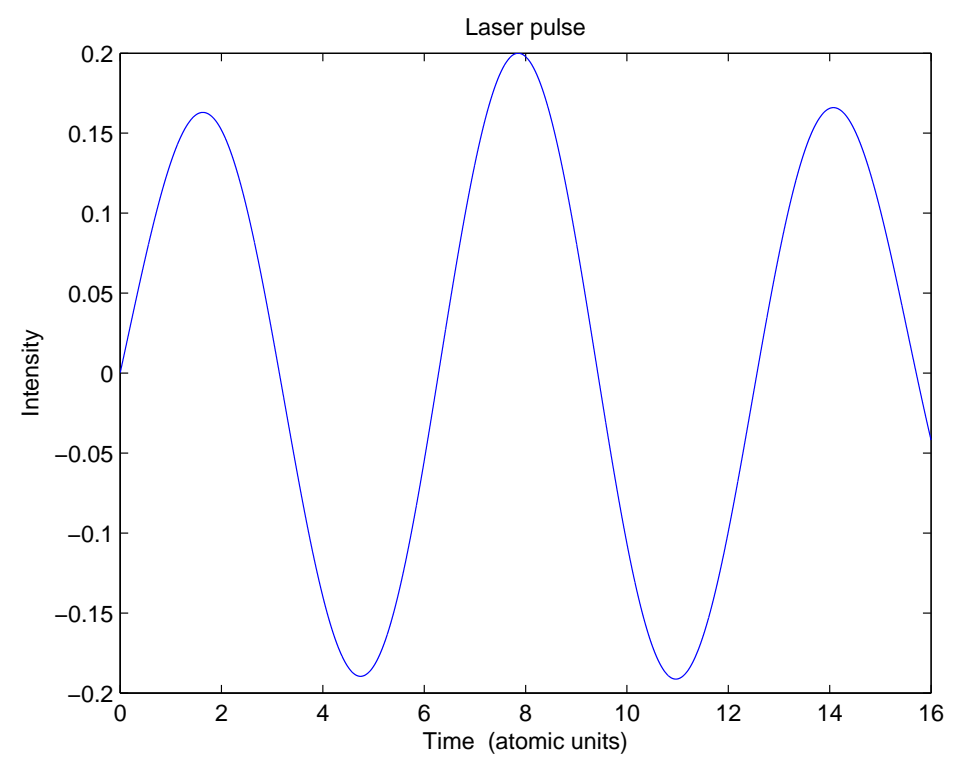

Figure 12: Laser pulse as a function of time.

time $t=7.7$ and $t=12.8$, in Fig. 20 (resp. time $t=20.5$, and $t=30.7$ in Fig. 21) the wavefunction of reference computed on the fine mesh $\psi_{\text {ref }}^{h_{\varepsilon}}$, the low/moderate frequency contribution computed on the coarse mesh $\psi_{\mathrm{Q}}^{h}$, and the reconstructed wavefunction on the fine mesh, $\psi^{h_{\varepsilon}}$, from $\psi_{\mathrm{FGA}}^{h_{\varepsilon}}$ and $\mathcal{L}_{h \rightarrow h_{\varepsilon}} \psi_{\mathrm{Q}}^{h}$. The spatial spectrum of i) the Cauchy data $\phi_{0}$, ii) the wavefunction of reference $\psi_{\text {ref }}^{h_{\varepsilon}}$ and of the reconstructed 
Figure 13: Comparison between i) the Cauchy data of reference on the fine mesh, ii) the reconstructed Cauchy data from the FGA and the Cauchy data on the coarse mesh, iii) the Cauchy data on the coarse mesh, and iv) the FGA contribution. (Up) Real part. (Down) Imaginary part.

solution $\psi^{h_{\varepsilon}}$ are represented in Fig. 24 at time $t=7.7$ and $t=12.8$ (resp. at time $t=20.5$ and $t=30.7$ in Fig. 25). In addition, we compare in Fig. 22 at time $t=7.7$ and $t=12.8$ (resp. time $t=20.5$ and $t=30.7$ in Fig. 23) the high frequency contribution (frequencies above $\eta^{-1}$ ) of the solution of reference $\psi_{\text {ref }}^{h_{\varepsilon}}$ with $\psi_{\mathrm{FGA}}^{h_{\varepsilon}}$. These results show the very good accuracy of the proposed reconstruction (coarse mesh+FGA), when high harmonic generation occurs. 
Figure 14: Comparison at time $T=16$ between i) the wavefunction of reference on the fine mesh, ii) the reconstructed wavefunction on the fine mesh from the FGA and the wavefunction on the coarse mesh, iii) the wavefunction on the coarse mesh, and iv) the FGA contribution. (Up) Real part. (Down) Imaginary part.

\section{Concluding remarks}

\subsection{Multilevel method for multi-high-frequency regime}

The two-level method which was presented in this paper, can easily be extended to a multilevel method, by considering multiple FGAs. More specifically, we consider a decreasing and real finite sequence $\left\{\varepsilon_{i}\right\}_{0 \leqslant i \leqslant L}$ associated to increasing frequencies $\left\{\varepsilon_{i}^{-1}\right\}_{0 \leqslant i \leqslant L}$. As before the contribution of frequencies smaller than $\varepsilon_{0}^{-1}$ (which was denoted by $\varepsilon^{-1}$ in Section 3.2 ) is computed by solving (1) in the quantum regime and on a coarse 
Figure 15: Spectrum of the Cauchy data, of the wavefunction of reference on the fine mesh, and of the reconstructed wavefunction on the fine mesh, at final time $T=16$.

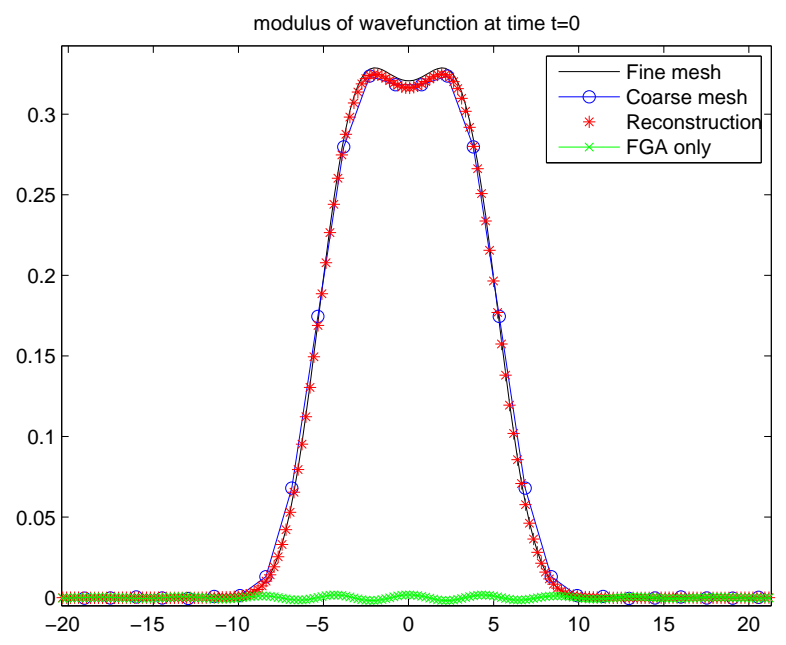

Figure 16: i) Groundstate of reference on the fine mesh, ii) reconstructed groundstate from the FGA and the groundstate on the coarse mesh, iii) groundstate on the coarse mesh, iv) and the FGA contribution.

mesh. By Fourier filtering, we construct a finite sequence of FGAs, $\left\{\psi_{\mathrm{FGA}}^{h_{\varepsilon_{i}}}\right\}_{1 \leqslant i \leqslant L}$ respectively associated to the frequency ranges $\left(\left(\varepsilon_{i-1}^{-1}+\varepsilon_{i}\right) / 2,\left(\varepsilon_{i}^{-1}+\varepsilon_{i+1}\right) / 2\right)$, for $i \geqslant 1$. We then follow the same strategy as in Sections 3.2 and 3.3 . 
Figure 17: Spatial frequency of i) the exact groundstate, ii) the groundstate of reference on the fine mesh, and iii) the reconstructed groundstate.
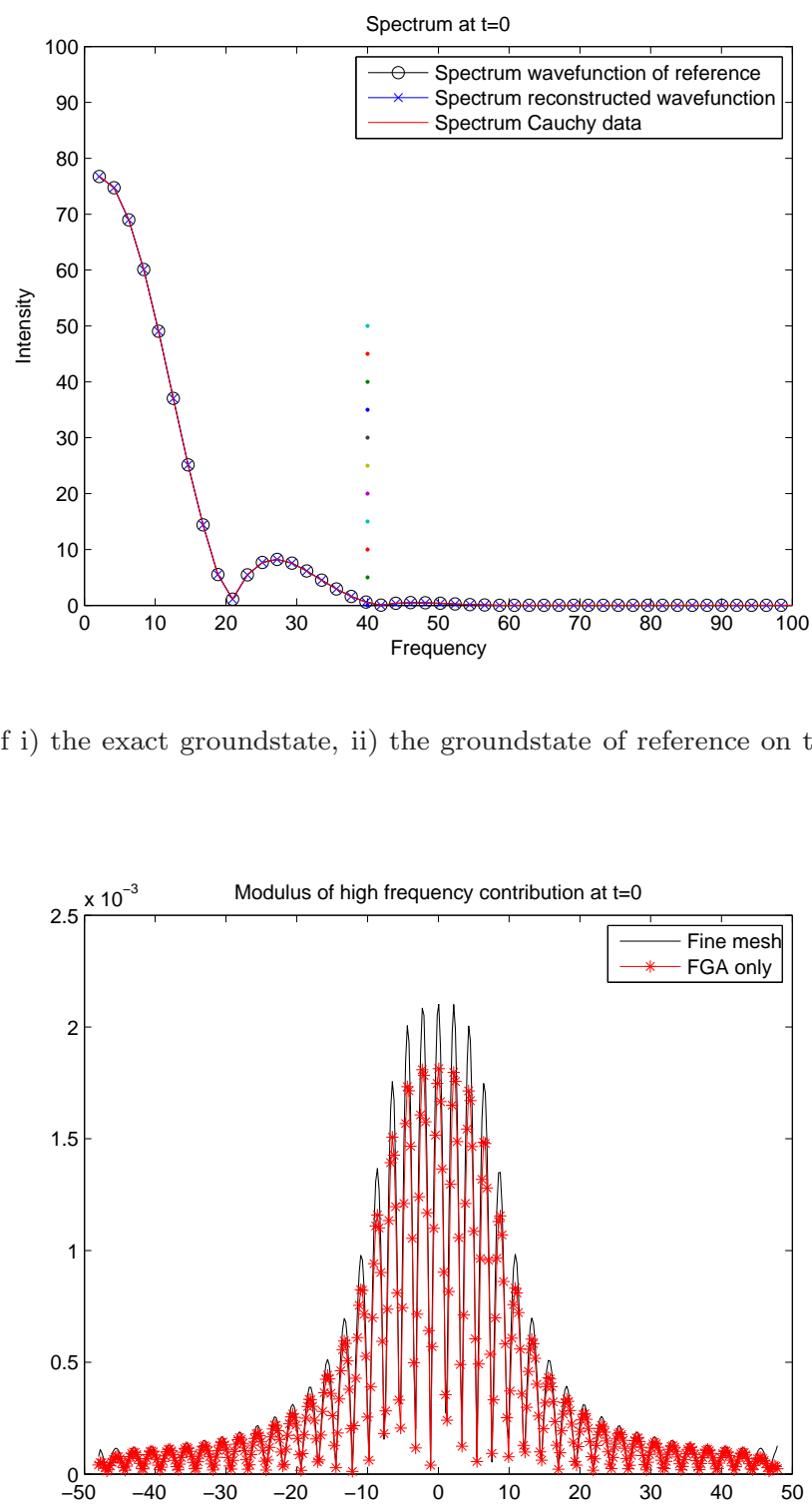

Figure 18: High frequency contribution: i) groundstate on the fine mesh, and ii) FGA contribution.

\subsection{Conclusion}

In this paper, we have derived two two-level algorithms for the numerical computation of the TDSE in wide frequency regimes, with and without high frequency generation. The strength of the method is that it allows for the efficient computation of wide frequency range solutions using a coarse mesh approximation for low/moderate frequencies and a semi-classical approximation, the Frozen Gaussian Approximation, for the high frequencies. Accurate numerical results were shown in both cases. A forthcoming paper will be dedicated to extended and more realistic tests and the optimization of the algorithms, in the framework of intense and short laser-molecule interaction, where high order harmonic generation occur. 
Figure 19: As a function of time, and using classical mechanics: (Up) Electric field and recombination times. (Middle) Electronic position. (Down) Kinetic energy.
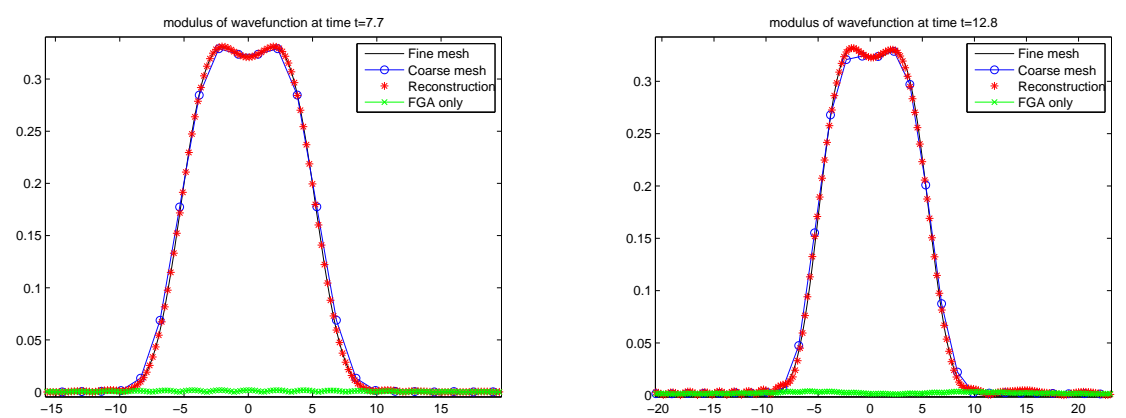

Figure 20: At time $t=7.7$ and $t=12.8$ : i) wavefunction of reference on the fine mesh, ii) reconstructed wavefunction on the fine mesh from the FGA and the wavefunction on the coarse mesh, iii) wavefunction on the coarse mesh, iv) FGA contribution.

\section{Acknowledgements.}

E. Lorin authors thanks NSERC for the financial support via the Discovery Grant program. X. Yang was partially supported by the NSF grants DMS-1418936 and DMS-1107291, and Hellman Family Foundation Faculty Fellowship, UC Santa Barbara. 

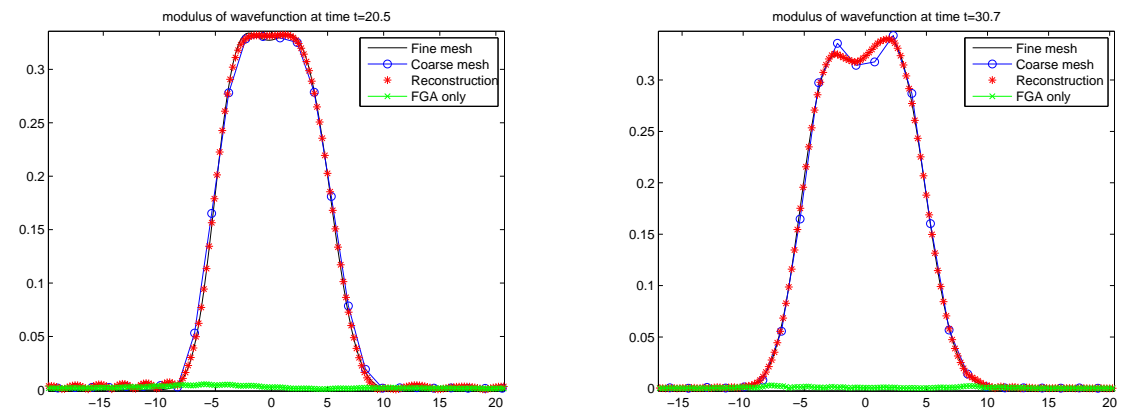

Figure 21: At time $t=20.5$ and $t=30.7$ : i) wavefunction of reference on the fine mesh, ii) reconstructed wavefunction on the fine mesh from the FGA and the wavefunction on the coarse mesh, iii) wavefunction on the coarse mesh, iv) FGA contribution.
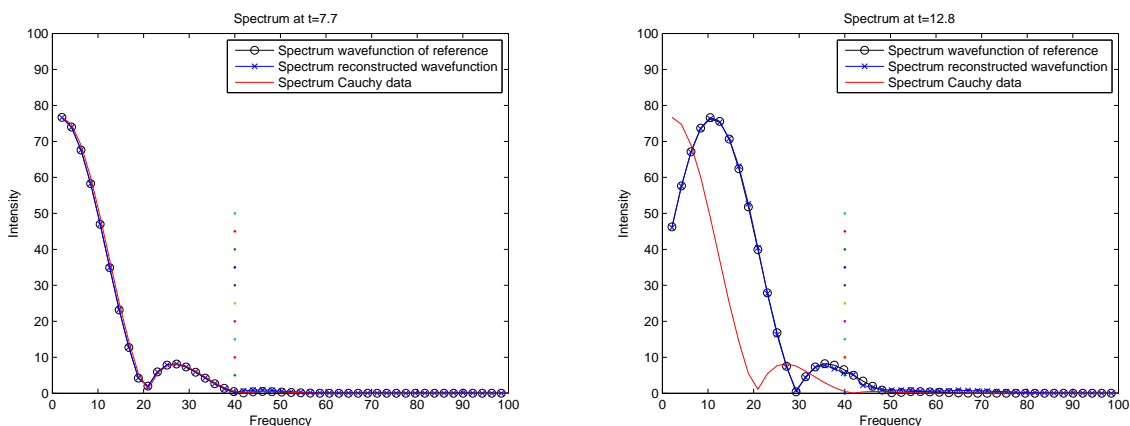

Figure 22: Spatial frequency at time $t=7.7$ and $t=12.8$ of i) the exact groundstate, ii) the wavefunction of reference on the fine mesh, and iii) the reconstructed wavefunction on the fine mesh.
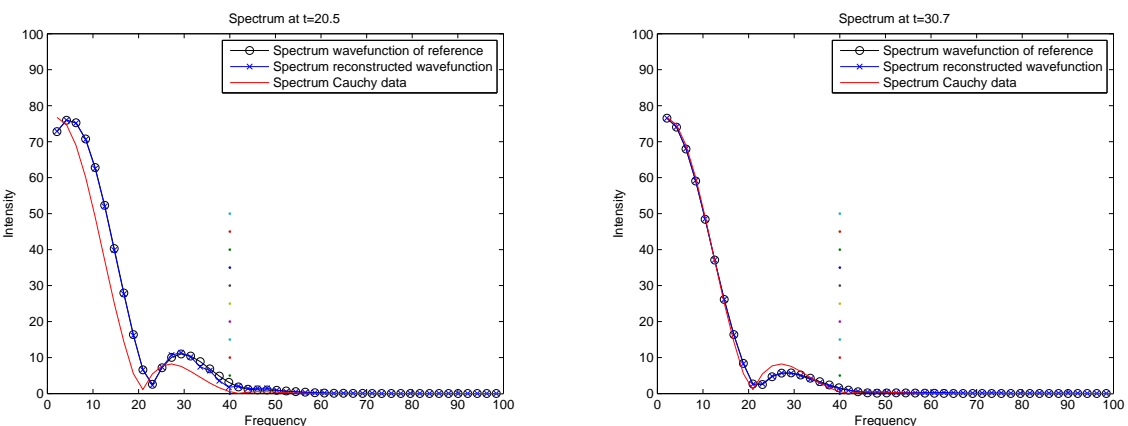

Figure 23: Spatial frequency at time $t=20.5$ and $t=30.7$ of i) the exact groundstate, ii) the wavefunction of reference on the fine mesh, and iii) the reconstructed wavefunction on the fine mesh.

\section{References}

[1] A. D. Bandrauk, S. Chelkowski, D. J. Diestler, J. Manz, and K.-J. Yuan. Quantum simulation of high-order harmonic spectra of the hydrogen atom. Phys. Rev. A, 79:023403, 2009.

[2] A.D. Bandrauk. Molecules in laser fields. In E. Yurtsever, editor, Frontiers of Chemical Dynamics, 

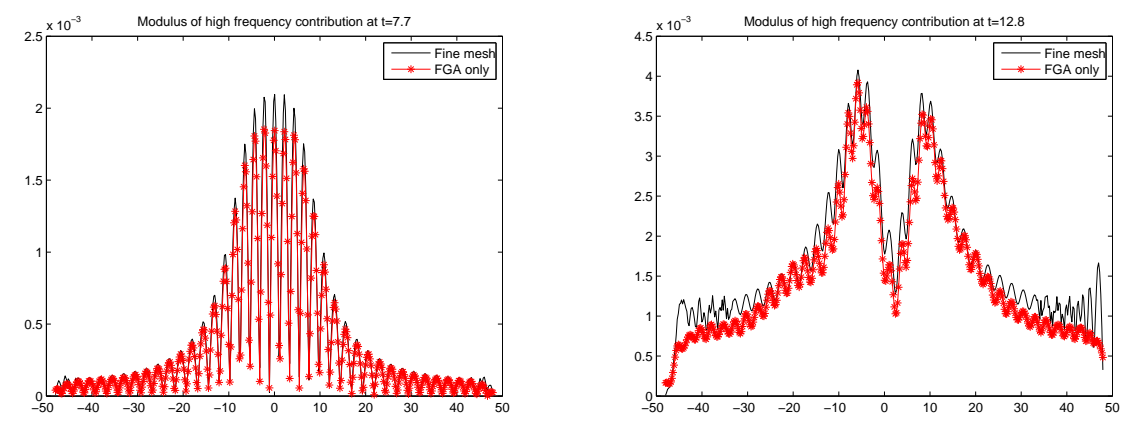

Figure 24: High frequency contribution at time $t=7.7$ and $t=12.8$ : i) reconstructed wavefunction on the fine mesh, and ii) FGA contribution.
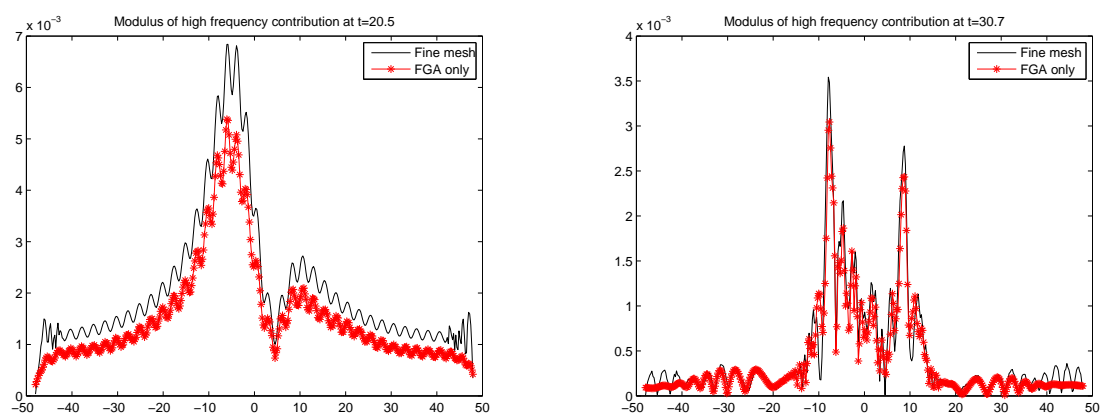

Figure 25: High frequency contribution at time $t=20.5$ and $t=30.7$ : i) reconstructed wavefunction on the fine mesh, and ii) FGA contribution.

volume 470 of NATO ASI Series, pages 131-150. Springer Netherlands, 1995.

[3] A.D. Bandrauk, F. Fillion-Gourdeau, and E. Lorin. Atoms and molecules in intense laser fields: Gauge invariance of theory and models. Journal of Physics B: Atomic, Molecular and Optical Physics, 46(15), 2013.

[4] C. Cohen-Tannoudji, J. Dupont-Roc, and G. Grynberg. Photons and atoms. Introduction to quantum electrodynamics. John Wiley and Sons Ltd, 1997.

[5] P.B. Corkum and F. Krausz. Attosecond science. Nature Physics, 3(6):381-387, 2007.

[6] B. Engquist and O. Runborg. Computational high frequency wave propagation. Acta Numer., 12:181$266,2003$.

[7] M.F. Herman and E. Kluk. A semiclasical justification for the use of non-spreading wavepackets in dynamics calculations. Chemical Physics, 91(1):27-34, 1984.

[8] S. Jin, P.A. Markowich, and C. Sparber. Mathematical and computational methods for semiclassical Schrödinger equations. Acta Numer., 20:211-289, 2011.

[9] S. Jin, H. Wu, and X. Yang. Gaussian beam methods for the Schrödinger equation in the semi-classical regime: Lagrangian and Eulerian formulations. Commun. Math. Sci., 6:995-1020, 2008.

[10] M. Lewenstein, Ph. Balcou, M.Yu. Ivanov, A. L'Huillier, and P.B. Corkum. Theory of high-harmonic generation by low-frequency laser fields. Phys. Rev. A, 49(3):2117-2132, 1994. 
[11] E. Lorin, S. Chelkowski, and A. Bandrauk. Attosecond pulse generation from aligned molecules dynamics and propagation in $\mathrm{H}_{2}^{+}$. New J. Phys., 10(025033):21pp, 2008.

[12] E. Lorin, X. Yang, and X. Antoine. Frozen gaussian approximation based domain decomposition methods for the linear schrdinger equation beyond the semi-classical regime. J. of Comput. Phys., 315:221$237,2016$.

[13] J. Lu and X. Yang. Frozen Gaussian approximation for high frequency wave propagation. Commun. Math. Sci., 9(3):663-683, 2011.

[14] J. Lu and X. Yang. Convergence of frozen Gaussian approximation for high-frequency wave propagation. Comm. Pure Appl. Math., 65(6):759-789, 2012.

[15] J. Lu and X. Yang. Frozen Gaussian approximation for general linear strictly hyperbolic systems: formulation and Eulerian methods. Multiscale Model. Simul., 10(2):451-472, 2012.

[16] P. Markowich, P. Pietra, and C. Pohl. Numerical approximation of quadratic observable of Schrödinger equation-type equations in the semiclassical limit. Numer. Math., 81:595-630, 1999.

[17] T. Swart and V. Rousse. A mathematical justification for the Herman-Kluk propagator. Comm. Math. Phys., 286(2):725-750, 2009.

[18] X. Yang and J. Zhang. Computation of the Schrödinger equation in the semiclassical regime on an unbounded domain. SIAM J. Numer. Anal., 52(2):808-831, 2014. 